TITLE:

\title{
Total Synthesis of $(+)$-Conolidine by the Gold(I)-Catalyzed Cascade Cyclization of a Conjugated Enyne
}

$\operatorname{AUTHOR}(\mathrm{S})$ :

Naoe, Saori; Yoshida, Yusuke; Oishi, Shinya; Fujii, Nobutaka; Ohno, Hiroaki

\section{CITATION:}

Naoe, Saori ... [et al]. Total Synthesis of (+)-Conolidine by the Gold(I)-Catalyzed Cascade Cyclization of a Conjugated Enyne. The Journal of Organic Chemistry 2016, 81(13): 56905698

\section{ISSUE DATE:}

2016-07

URL:

http://hdl.handle.net/2433/241631

\section{RIGHT:}

This document is the Accepted Manuscript version of a Published Work that appeared in final form in 'The Journal of Organic Chemistry', copyright @ American Chemical Society after peer review and technical editing by the publisher. To access the final edited and published work see https://doi.org/10.1021/acs.joc.6b00720.; The full-text file will be made open to the public on $8 \mathrm{June} 2017$ in accordance with publisher's 'Terms and Conditions for Self-Archiving'.; この論文は 出版社版でありません。引用の際には出版社版をご確認ご利用ください。; This is not the published version. Please cite only the published version. 


\title{
Total Synthesis of (+)-Conolidine by the Gold(I)-
}

\section{Catalyzed Cascade Cyclization of a Conjugated Enyne}

\author{
Saori Naoe, Yusuke Yoshida, Shinya Oishi, Nobutaka Fujii,* and Hiroaki Ohno*
}

Graduate School of Pharmaceutical Sciences, Kyoto University, Sakyo-ku, Kyoto 606-8501, Japan hohno@pharm.kyoto-u.ac.jp; nfujii@pharm.kyoto-u.ac.jp

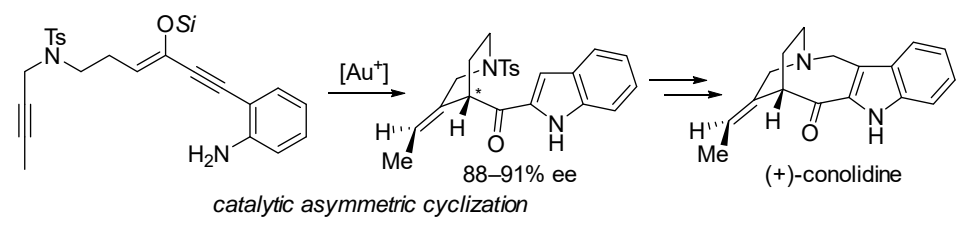

\begin{abstract}
A total synthesis of (+)-conolidine has been achieved via the gold(I)-catalyzed cascade cyclization of a conjugated enyne. Remarkably, this strategy allowed for the simultaneous formation of the indole ring and the ethylidene-substituted piperidine moiety of $(+)$-conolidine under homogenous gold catalysis in an enantioselective manner (88-91\% ee).
\end{abstract}

Conolidine (1), which belongs to the C5-nor stemmadenine family of alkaloids, was first isolated from Tabernaemonta divaricata by Kam et al. in 2004 (Figure 1). ${ }^{1}$ This group only managed to isolate $0.0013 \mathrm{~g}$ of conolidine from the stem bark of this small flowering plant. Since Bohn, Micalizio, and co-workers accomplished the first asymmetric total synthesis of conolidine (1) in 2011, there has been considerable interest in its unique analgesic activity, which differs from that of many common opioids, including morphine. ${ }^{2}$ Although several efficient methods have been reported for the synthesis of C5-nor stemmadenine-type indoles, ${ }^{2-4}$ the development of a diversityoriented route suitable for evaluating the structure-activity relationships of these compounds is still highly desired. 

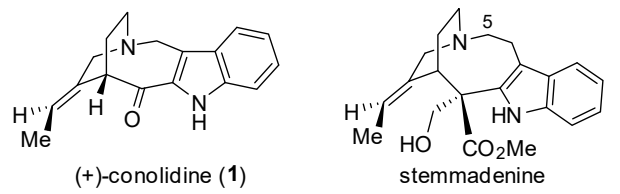

Figure 1. Stemmadenine-based alkaloids

Homogeneous gold catalysis has attracted considerable attention because of the strong $\pi$ acidity of gold, as well as its potential to stabilize cationic reaction intermediates. ${ }^{5}$ The versatile reactivity of gold catalysts has allowed for the design of several eloquent cascade reactions for the direct step- and atom-economical synthesis of complex molecules. ${ }^{6}$ Nowadays, homogeneous gold catalysis is recognized as one of the most effective strategies for the electrophilic activation of alkynes for the synthesis of natural products. ${ }^{6 f}$

We recently reported the gold(I)-catalyzed bis-cyclization of conjugated diynes $2\left(\mathrm{R}^{\prime}=\mathrm{H},-\right.$ $\mathrm{NuH}=\mathrm{CH}_{2} \mathrm{OH}$ ) as an efficient strategy for the construction of fused indoles 3 and 4 (Scheme 1). ${ }^{7}$ In this reaction, the initial indole formation occurred via a 5-endo-dig cyclization, which was followed by a 7-endo-dig cyclization to give the fused indole $\mathbf{3}$ as the major product. Based on this reaction, we designed a strategy for the synthesis of $(+)$-conolidine (1) (Scheme 2, strategy I). It was envisaged that the known conolidine precursor $7^{2,4}$ could be prepared by the gold(I)-catalyzed cascade cyclization of conjugated diyne 10. In this sense, the bis-cyclization reaction would allow for the formation of a fused indole (path a and b), which would be followed by a third cyclization to give the piperidine moiety. One of the potential issues with this strategy would be controlling the regioselectivity of the second cyclization step. In particular, the 6-exo-dig cyclization (path b) would need to be favored over the 7-endo-dig pathway (path b') to allow for the introduction of the oxygen atom at the appropriate carbon of the product. We also designed a second strategy (strategy II) using a conjugated enyne $\mathbf{1 1}$ bearing a silyl enol ether. ${ }^{8}$ Notably, this strategy would avoid the need to control the regioselectivity of the second cyclization described in strategy I by introducing the oxygen atom of the conolidine as a silyl ether. It was also envisaged that the nucleophilicity of 
the enol ether would be increased following the formation of the indole, leaving it better equipped to promote the subsequent formation of the piperidine ring. Furthermore, the use of a chiral auxiliary (in strategy I) or chiral gold complex would allow for the asymmetric induction of these key steps. Both of these strategies involve the use of readily accessible synthons 12-16, and can therefore be considered as diversity-oriented convergent syntheses. Herein, we report the total synthesis of $(+)$-conolidine (1) based on the catalytic asymmetric cyclization of the enol ether-type substrate 11 (strategy II).

Scheme 1. Previous Work: Gold(I)-Catalyzed Intra-molecular Consecutive Cyclizations of a Conjugated Diyne

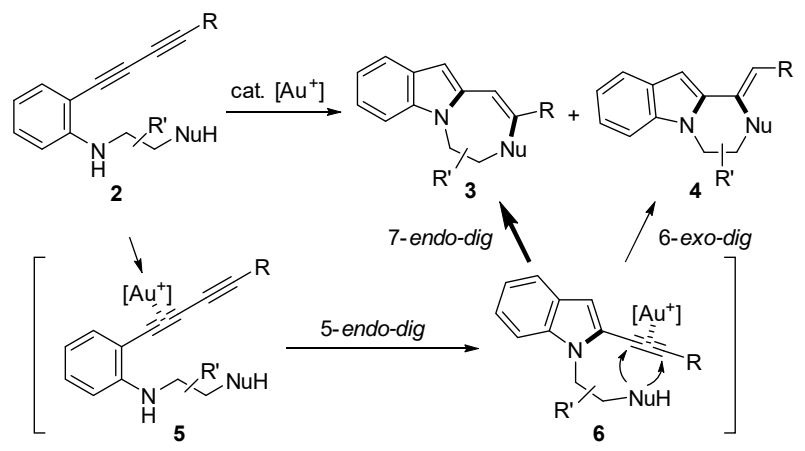

Scheme 2. Retrosynthetic Analysis of Conolidine Based on the Gold(I)-Catalyzed Cascade Reaction of Conjugated Alkynes 

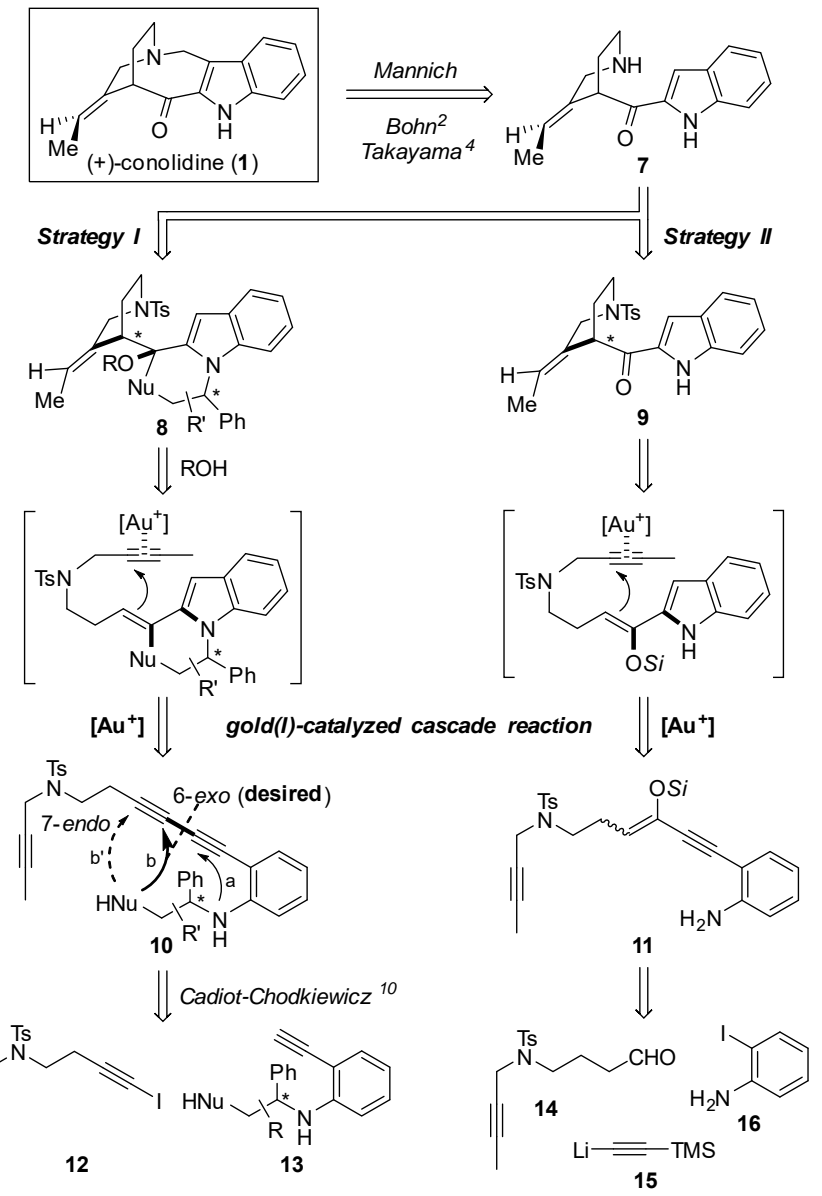

Strategy I. We initially conducted a series of model experiments using conjugated diynes $\mathbf{2 a}-\mathbf{c}$ to evaluate the possibility of controlling the regioselectivity in strategy I (Scheme 3). The reaction of $2 \mathbf{a}$ bearing a phenylglycinol moiety gave the desired isomer $\mathbf{4 a}$ as a minor product $(\mathbf{4 a} / \mathbf{3 a}=$ 29:71). In contrast, substrate $\mathbf{2 b}$ bearing a vicinal phenyl group and substrate $\mathbf{2 c}$ bearing a carboxylic acid preferentially afforded the corresponding 6-exo-products $(4 / 3=71: 29-80: 20)$. Based on these results, we prepared the corresponding alcohol and carboxylic acid substrates 10a and $\mathbf{1 0 b}$ as the most suitable candidates for the synthesis of conolidine.

Scheme 3. Model Experiment for Control of the Regioselectivity in the Second Cyclization

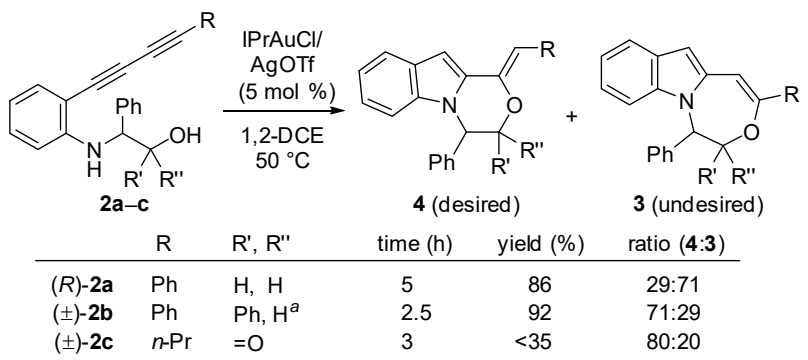


${ }^{a}$ The erythro-isomer of $( \pm)-\mathbf{2 b}$ was used.

Our initial efforts towards the preparation and subsequent gold(I)-catalyzed cyclization of the conjugated diynes $\mathbf{1 0 a}$ and $\mathbf{1 0 b}$ are shown in Scheme 4. The alkylation of tosylamide $\mathbf{1 7 ^ { 9 }}$ with 1bromobut-2-yne (18) gave diyne 19. The iodination of the terminal alkyne moiety in 19 with NIS and $\mathrm{AgNO}_{3}$, followed by the subsequent Cadiot-Chodkiewicz coupling ${ }^{10}$ of the resulting iodoalkyne with 13a or 13b gave the amino alcohol- and amino acid-type substrates $( \pm)-\mathbf{1 0 a}$ and 10b (after hydrolysis), respectively. Unfortunately, however, the subsequent reaction of 10a with IPrAuCl/AgOTf (10 mol \%) and EtOH (2 equiv) in 1,2-DCE at $50{ }^{\circ} \mathrm{C}$ for $2 \mathrm{~h}$ gave a complex mixture of unidentified products. In contrast, the reaction of $\mathbf{1 0 b}$ under the same conditions led to the formation of the bis-cyclization products $\mathbf{2 0 b}$ and $\mathbf{2 0 b}$ ' with good regioselectivity for the former of these two products $\left(\mathbf{2 0 b} / \mathbf{2 0} \mathbf{b}^{\prime}=90 / 10\right)$. It is noteworthy, however, that these compounds were formed in low yields $(<31 \%)$ because of their poor stability. Disappointingly, all of our other attempts to promote the formation of the piperidine using $\mathbf{1 0 b}$ and $\mathbf{2 0 b} / \mathbf{2 0 b}$ ' resulted in failure, most likely because of the poor nucleophilicity of the enol ether moiety of $\mathbf{2 0 b}$ bearing an electronwithdrawing group. Based on these results, we discarded strategy I and focused our efforts on strategy II using the conjugated enynes 11a and $\mathbf{1 1 b .}$

Scheme 4. Unsuccessful Attempts at the Gold(I)-Catalyzed Cyclization of the Conjugated Diynes 10a and 10b (Strategy I) 


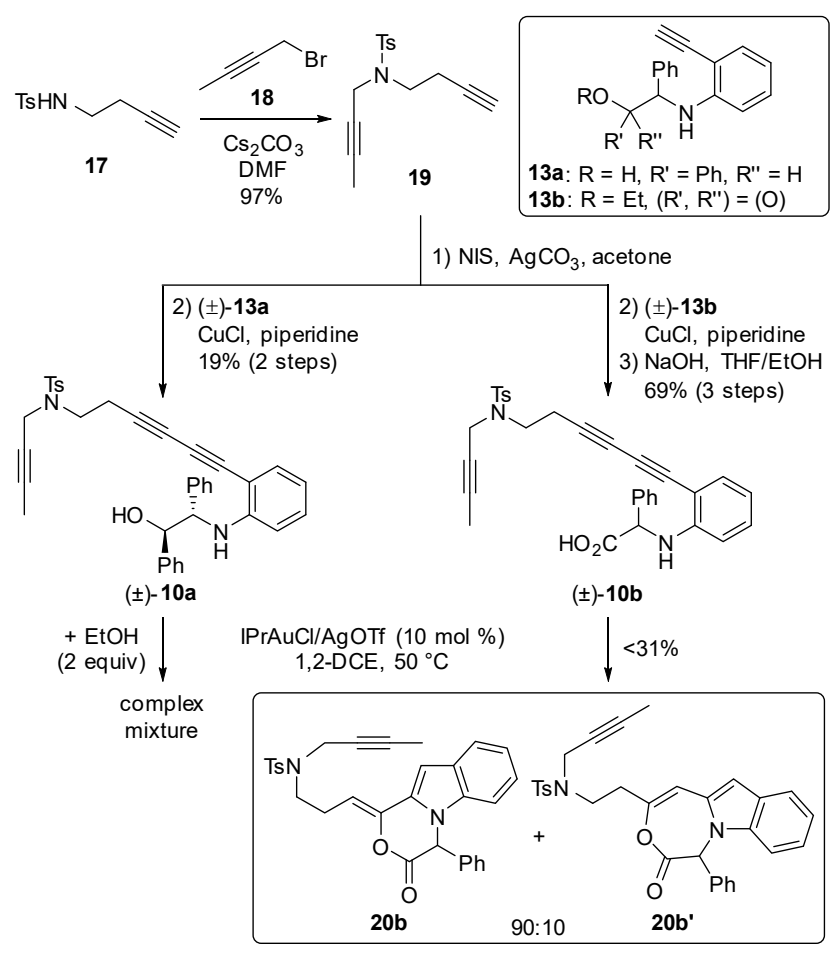

Strategy II. Conjugated enynes 11a and 11b bearing different silyl enol ether moieties were prepared according to route shown in Scheme 5. The alkylation of tosylamide $21^{11}$ with ethyl 4bromobutanoate (22) gave ester $\mathbf{2 3}$, which was reduced with DIBAL to give the corresponding aldehyde. The subsequent 1,2-addition of lithium (trimethylsilyl)acetylide (15) to this aldehyde, followed by the removal of the TMS group with TBAF afforded the terminal alkyne $\mathbf{2 5}$ in excellent yield. The Sonogashira coupling reaction of alkyne 25 with o-iodoaniline (16) provided alkynylaniline 26 in 90\% yield. The oxidation of $\mathbf{2 6}$ with $\mathrm{MnO}_{2}$ gave the corresponding ketone 27 in $71 \%$ yield, which was treated with TIPSOTf or TBSOTf in the presence of Et $3 \mathrm{~N}$ to give the conjugated enyne-type silyl enol ethers $\mathbf{1 1 a}$ and $\mathbf{1 1 b}$ in 75 and $81 \%$ yields, respectively. It is noteworthy that the $(E)$ - and (Z)-isomers ${ }^{12}$ of 11 could be separated, as necessary, by column chromatography over silica gel followed by PTLC (see Supporting Information).

Scheme 5. Preparation and Gold-Catalyzed Cyclization of the Conjugated Enynes 11a and 11b 

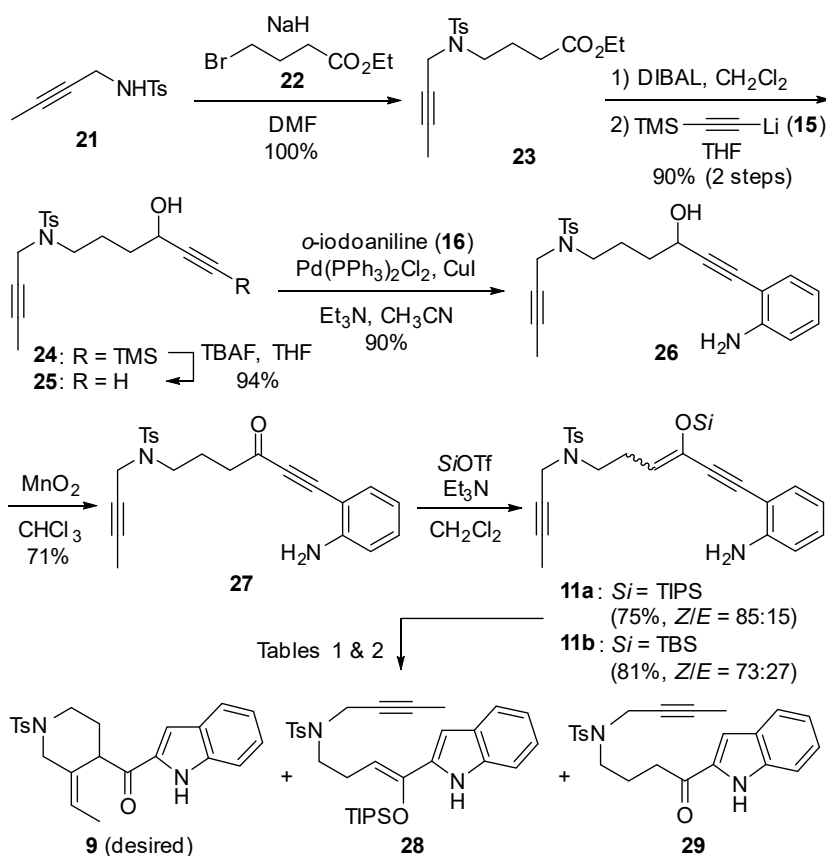

We then investigated the gold(I)-catalyzed cascade reaction of the enol ether-type conjugated enynes 11a and 11b (Table 1). The treatment of enyne 11a with $\mathbf{L 1 A u}(\mathrm{MeCN}) \mathrm{SbF}_{6}(5 \mathrm{~mol} \%)$ (Figure 2) in toluene- $d_{8}$ at room temperature afforded the desired product $9(16 \%)$, as well as the two monocyclization products $28^{13}$ (34\%) and 29 (14\%). To drive the reaction to completion, we investigated the use of an additive as a proton source as well as silyl scavenger. Fortunately, the addition of $\mathrm{H}_{2} \mathrm{O}^{4}$ improved the yields of 9 to $38 \%$ (entry 2), In contrast, the use of $\mathrm{MeOH}$ was less efficient (entry 3). The use of an IPr ligand was found to be unsuitable for this reaction (entry 4). Similarly, several other experiments using $\mathrm{NaBARF}^{8 \mathrm{~h}}$ (Figure 2) as the counter anion (entry 5), $\mathrm{CD}_{2} \mathrm{Cl}_{2}$ as a solvent (entry 6 ) or the TBS ether $\mathbf{1 1 b}$ as a substrate (entry 7 ) did not improve the yield.

Table 1. Optimization of the Reaction Conditions ${ }^{a}$

\begin{tabular}{|c|c|c|c|c|c|c|c|}
\hline \multirow{2}{*}{ entry } & \multirow{2}{*}{ Ligand } & \multirow{2}{*}{ additive } & \multirow{2}{*}{$\mathrm{R}$} & \multirow{2}{*}{ time (h) } & \multicolumn{3}{|c|}{ yield $(\%)^{b}$} \\
\hline & & & & & 9 & 28 & 29 \\
\hline 1 & L1 & - & TIPS & 24 & 16 & 34 & 14 \\
\hline 2 & L1 & $\mathrm{H}_{2} \mathrm{O}$ & TIPS & 24 & 38 & - & 2 \\
\hline 3 & $\mathbf{L 1}$ & $\mathrm{MeOH}$ & TIPS & 19 & 29 & - & 2 \\
\hline 4 & $\mathrm{IPr}$ & $\mathrm{H}_{2} \mathrm{O}$ & TIPS & 24 & 3 & 45 & 10 \\
\hline
\end{tabular}




$\begin{array}{llllllll}5 & \mathbf{L 1}^{c} & \mathrm{H}_{2} \mathrm{O} & \text { TIPS } & 24 & 15 & 5 & 45 \\ 6^{d} & \mathbf{L 1} & \mathrm{H}_{2} \mathrm{O} & \text { TIPS } & 24 & 16 & - & 43 \\ 7 & \mathbf{L 1} & \mathrm{H}_{2} \mathrm{O} & \text { TBS } & 24 & 33 & - & -\end{array}$

${ }^{a}$ Unless otherwise noted, all of these reactions were carried out using 11a $(Z / E=79: 21)$ or 11b $(\mathrm{Z} / E=71: 29)$ with $\mathbf{L} 1 \mathrm{Au}(\mathrm{MeCN}) \mathrm{SbF}_{6}$ or $\operatorname{IPrAuCl}(5 \mathrm{~mol} \%) / \mathrm{AgSbF}_{6}(5 \mathrm{~mol} \%)$ in toluene-d $(0.2$ M) at room temperature in the presence of an additive (1.5 equiv). ${ }^{b}$ NMR yields were evaluated using mesitylene as an internal standard. ${ }^{c}$ Using $\mathbf{L 1 A u C l} / \mathrm{NaBARF} .{ }^{d}$ Using $\mathrm{CD}_{2} \mathrm{Cl}_{2}$ as a solvent instead of toluene- $d 8$.

Figure 2. Ligands and co-catalysts screened in this study

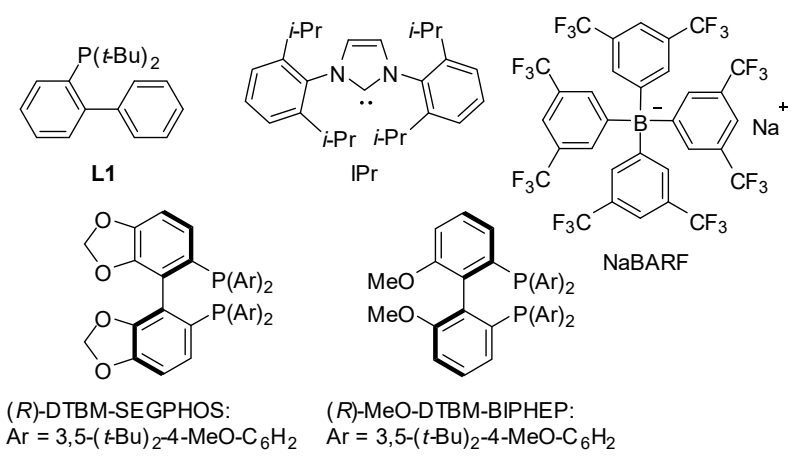

We then proceeded to investigate the enantioselective gold(I)-catalyzed cascade reaction of the conjugated enyne 11a (Table 2). Based on a related study reported by Toste and co-workers involving the asymmetric carbocyclization of a silyl enol ether, ${ }^{8 \mathrm{~h}}$ we investigated the use of biarylphosphine-type dinuclear chiral gold complexes to affect this reaction (Figure 2). The treatment of the conjugated enyne 11a with $(R)$-DTBM-SEGPHOS(AuCl $)_{2}(5 \mathrm{~mol} \%) / \mathrm{AgSbF}_{6}(10$ mol \%) resulted in the formation of the undesired ketone $\mathbf{2 9}$ as the major product (entry 1). The use of $(R)$-MeO-DTBM-BIPHEP gave the desired product (S)-9 in 13\% yield and $89 \%$ ee (entry 2 ). An increase in catalyst loading ( $10 \mathrm{~mol} \%$ for the bimetallic gold complex) led to a slight decreased in the yield to $10 \%$, as well as a decrease in the ee to $76 \%$ (entry 3 ). Expecting that the sterically less hindered $(Z)$-isomer has better reactivity, we examined the reaction of the both isomers, $(Z)$ - and (E)-11a. Interestingly, the use of (Z)-11a led to an improvement in the yield of $(S)-9$ to $32 \%$ (entry 5), whereas the reaction of $(E)$-11a failed to afford the desired product (entry 4). Taken together, 
these results suggested that it was only possible to generate the desired product 9 from the $Z$-isomer of 11a when a $E / Z$ mixture of 11a was used as the substrate (entries 1-3). The use of 11a in conjunction with a decreased loading of $\mathrm{H}_{2} \mathrm{O}$ (1.0 equiv) led to an improvement in the ee to $91 \%$, although the yield dropped to $18 \%$ (entry 6 ).

\section{Table 2. Enantioselective Gold(I)-Catalyzed Cyclization ${ }^{a}$}

\begin{tabular}{llllll}
\multirow{2}{*}{ entry } & $\begin{array}{l}Z / E \\
(\mathbf{1 1})\end{array}$ & catalyst & $\begin{array}{l}\text { time } \\
\text { yield of }\end{array}$ & $\begin{array}{l}\text { \% ee } \\
\mathbf{9}(\%)^{b}\end{array}$ & {$[(S)-9]$}
\end{tabular}

${ }^{a}$ Unless otherwise noted, these reactions were carried out using 11a in toluene $(0.2 \mathrm{M})$ at room temperature in the presence of $\mathrm{H}_{2} \mathrm{O}$ (1.5 equiv) with a catalyst loading of 5 (for the bimetallic gold complex) and $10 \mathrm{~mol} \%$ (for $\mathrm{AgSbF}_{6}$ ). ${ }^{b}$ Isolated yields. ${ }^{c}$ Determined by chiral HPLC. ${ }^{d}$ Ketone 29 was obtained as the major product. ${ }^{e}$ N.D. $=$ not detected. ${ }^{f}$ The catalyst loading was increased to 10 and $20 \mathrm{~mol} \mathrm{\% .}{ }^{g}$ Using $\mathrm{H}_{2} \mathrm{O}$ (1.0 equiv).

Finally, we investigated the conversion of the bis-cyclization product $(S)-9(91 \%$ ee) to $(+)-$ conolidine (1). The treatment of $(S)-9$ with $\mathrm{Na} /$ naphthalene resulted in the cleavage of the Ts protecting group to give the known conolidine precursor 7 in $60 \%$ yield (Scheme 6). According to the procedure reported by Bohn, Micalizio, and co-workers, ${ }^{2}$ we obtained $(+)$-conolidine (1) in $34 \%$ yield and $84 \%$ ee. The spectroscopic and specific optical rotation data for the synthetic conolidine were identical to those reported in the literature. ${ }^{1,2}$

Scheme 6. Total Synthesis of $(+)$-Conolidine 


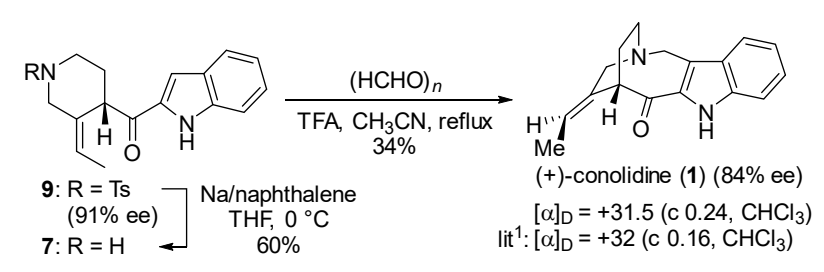

In conclusion, we have achieved the total synthesis of $(+)$-conolidine based on the gold(I)catalyzed cascade cyclization of a conjugated enyne. This study has shown that the feasibility of catalytic asymmetric reactions involving chiral gold(I) complexes for the construction of stemmadenine-type scaffolds.

\section{Experimental Section}

General Methods. For open column chromatography, silica gel or $\mathrm{NH}_{2}$ silica gel was employed. Thin layer chromatography was performed on TLC silica gel $60 \mathrm{~F}_{254}$ or $\mathrm{NH}_{2}$ silica gel 60 F254 plate (layer thickness $0.25 \mathrm{~mm}$ ), which were developed using standard visualizing agents: UV fluorescence $(254 \mathrm{~nm})$ and anisaldehyde with heating. Melting points were measured by a hot stage melting point apparatus (uncorrected). In ${ }^{1} \mathrm{H}$ NMR spectra, chemical shifts are reported in $\delta(\mathrm{ppm})$ relative to TMS as internal standard. In ${ }^{13} \mathrm{C}$ NMR spectra, chemical shifts are referenced to the residual solvent signal. ${ }^{1} \mathrm{H}$ NMR spectra are tabulated as follows: chemical shift, multiplicity $(\mathrm{b}=$ broad, $\mathrm{s}=$ singlet, $\mathrm{d}=$ doublet, $\mathrm{t}=$ triplet, $\mathrm{q}=$ quartet, $\mathrm{m}=$ multiplet), number of protons, and coupling constant(s).

The compounds 16, 18, 22, and (R)-DTBM-SEGPHOS $(\mathrm{AuCl})_{2}$ were obtained commercially and used without further purification. The known compounds $\mathbf{S 1},{ }^{14} \mathbf{S 4},{ }^{15} \mathbf{S} 7,{ }^{16} \mathbf{1 7},{ }^{9} \mathbf{2 1},{ }^{11}$ and $(R)-$ MeO-BIPHES(AuCl) ${ }_{2}^{17,18}$ were prepared according to the literature. Structures of $\mathbf{S 1 - S 9}$ are shown in Schemes S1-S3 (Supporting Information).

\section{Preparation of Starting Materials.}

(R)-2-Phenyl-2-(\{2-[(trimethylsilyl)ethynyl]phenyl\}amino)ethan-1-ol (S2). The coupling of 
S1 and trimethylsilylacetylene was carried out according to the reported method ${ }^{19}$ as follows: to a stirred suspension of $\mathbf{S 1}(1.42 \mathrm{~g}, 4.86 \mathrm{mmol}), \mathrm{PdCl}_{2}(\mathrm{PhCN}) 2(112 \mathrm{mg}, 0.29 \mathrm{mmol})$ and $\mathrm{CuI}$ (55.6 mg, $0.29 \mathrm{mmol})$ in dry 1,4-dioxane $(10 \mathrm{~mL})$ under argon were added diisopropylamine $(3.4 \mathrm{~mL}, 24.2$ $\mathrm{mmol})$, trimethylsilylacetylene $(0.7 \mathrm{~mL}, 5.06 \mathrm{mmol})$ and tri(tert-butyl)phosphine $(0.2 \mathrm{~mL}, 0.85$ mmol). After stirring at $50{ }^{\circ} \mathrm{C}$ for $12 \mathrm{~h}$, the reaction mixture was diluted with EtOAc and filtered through a short pad of silica gel. The filtrate was concentrated in vacuo and the residue was chromatographed on silica gel (hexane/EtOAc $=5 / 1)$ to afford $\mathbf{S 2}(976 \mathrm{mg}, 65 \%)$ as amber oil: $[\alpha]^{29}{ }_{\mathrm{D}} 242$ (c 0.51, $\left.\mathrm{CHCl}_{3}\right)$; IR (neat): $3393(\mathrm{OH}), 2143(\mathrm{C} \equiv \mathrm{C}) ;{ }^{1} \mathrm{H}$ NMR $\left(500 \mathrm{MHz}, \mathrm{CDCl}_{3}\right) \delta: 0.30$ (s, 9H), 1.69 (br s, 1H), 3.80-3.83 (br m, 1H), 3.96-4.00 (br m, 1H), 4.56-4.57 (br m, 1H), 5.52-5.53 (br m, 1H), $6.38(\mathrm{~d}, J=8.6 \mathrm{~Hz}, 1 \mathrm{H}), 6.57-6.58(\mathrm{~m}, 1 \mathrm{H}), 7.00-7.04(\mathrm{~m}, 1 \mathrm{H}), 7.25-7.35(\mathrm{~m}, 6 \mathrm{H}) ;{ }^{13} \mathrm{C}$ NMR (125 MHz, $\left.\mathrm{CDCl}_{3}\right) \delta: 0.0$ (3C), 59.3, 67.3, 100.7, 101.8, 108.0, 110.9, 116.6, 126.5 (2C), 127.6, 128.7 (2C), 129.9, 131.7, 139.7, 148.4; HRMS (FAB) calcd for $\mathrm{C}_{19} \mathrm{H}_{24} \mathrm{NOSi}\left(\mathrm{MH}^{+}\right)$ 310.1622 , found 310.1620 .

(R)-2-[(2-Ethynylphenyl)amino]-2-phenylethan-1-ol (S3). The desilylation of S2 was carried out according to the reported method ${ }^{20}$ as follows: $\mathrm{K}_{2} \mathrm{CO}_{3}(1.08 \mathrm{~g}, 8.0 \mathrm{mmol})$ was added to the solution of $\mathbf{S 2}(804 \mathrm{mg}, 2.60 \mathrm{mmol})$ in $\mathrm{MeOH}(26 \mathrm{~mL})$. After stirring at room temperature for $1 \mathrm{~h}$, the mixture was diluted with EtOAc. The organic layer was separated, washed with water and brine, dried over $\mathrm{MgSO}_{4}$, and concentrated in vacuo. The residue was chromatographed on silica gel (hexane/EtOAc $=5 / 1)$ to afford $\mathbf{S 3}(445 \mathrm{mg}, 72 \%)$ as pale amber powder: $\mathrm{mp} 79{ }^{\circ} \mathrm{C} ;[\alpha]^{26} \mathrm{D} 240(\mathrm{c}$ 1.06, $\left.\mathrm{CHCl}_{3}\right)$; IR (neat): $3401(\mathrm{OH}), 3253(\mathrm{C} \equiv \mathrm{CH}), 2089(\mathrm{C} \equiv \mathrm{C}) ;{ }^{1} \mathrm{H}$ NMR $\left(500 \mathrm{MHz}, \mathrm{CDCl}_{3}\right) \delta$ : $1.66(\mathrm{dd}, J=7.5,5.2 \mathrm{~Hz}, 1 \mathrm{H}), 3.50(\mathrm{~s}, 1 \mathrm{H}), 3.82-3.87(\mathrm{~m}, 1 \mathrm{H}), 3.97-4.03(\mathrm{~m}, 1 \mathrm{H}), 4.60(\mathrm{dd}, J=$ 10.4, $6.4 \mathrm{~Hz}, 1 \mathrm{H}), 5.47-5.48$ (br m, 1H), 6.39 (d, $J=8.7 \mathrm{~Hz}, 1 \mathrm{H}), 6.59-6.61$ (m, 1H), 7.04-7.06 (m, 1H), 7.27-7.30 (m, 1H), 7.31-7.38 (m, 5H); $\left.{ }^{13} \mathrm{C} \mathrm{NMR} \mathrm{(125} \mathrm{MHz,} \mathrm{CDCl}_{3}\right)$ 8: 59.4, 67.3, 80.6, 83.3, 106.9, 111.1, 116.8, 126.6 (2C), 127.7, 128.9 (2C), 130.2, 132.6, 139.6, 148.4; HRMS (FAB) calcd for $\mathrm{C}_{16} \mathrm{H}_{16} \mathrm{NO}\left(\mathrm{MH}^{+}\right)$238.1226, found 238.1232.

(R)-2-Phenyl-2-\{[2-(phenylbuta-1,3-diyn-1-yl)phenyl]amino\}ethan-1-ol (2a). The coupling 
of $\mathbf{S 3}$ and ethynylbenzene was carried out according to the reported method ${ }^{21}$ as follows: a mixture of $\mathbf{S 3}(432 \mathrm{mg}, 1.82 \mathrm{mmol})$, ethynylbenzene (1.0 mL, $9.11 \mathrm{mmol}), \mathrm{Cu}(\mathrm{OAc}) 2 \cdot \mathrm{H}_{2} \mathrm{O}(36.3 \mathrm{mg}, 0.18$ mmol) and piperidine $(0.5 \mathrm{~mL}, 5.06 \mathrm{mmol})$ in $\mathrm{CH}_{2} \mathrm{Cl}_{2}(9 \mathrm{~mL})$ was stirred in open atmospheric air at room temperature for $5 \mathrm{~h}$. The reaction mixture was concentrated in vacuo and the residue was chromatographed on silica gel (hexane/EtOAc $=5 / 1)$ to afford 2a $(349 \mathrm{mg}, 57 \%)$ as amber oil: $[\alpha]^{26}{ }_{\mathrm{D}} 470\left(\right.$ ( 1.00, $\left.\mathrm{CHCl}_{3}\right)$; IR (neat): $3391(\mathrm{OH}), 2208(\mathrm{C} \equiv \mathrm{C}), 2140(\mathrm{C} \equiv \mathrm{C}) ;{ }^{1} \mathrm{H}$ NMR $(500 \mathrm{MHz}$, $\left.\mathrm{CDCl}_{3}\right) \delta: 1.71-1.73(\mathrm{~m}, 1 \mathrm{H}), 3.86-3.89(\mathrm{~m}, 1 \mathrm{H}), 3.99-4.04(\mathrm{~m}, 1 \mathrm{H}), 4.60-4.62(\mathrm{~m}, 1 \mathrm{H}), 5.45-5.47$ (br m, 1H), 6.38 (d, $J=8.1 \mathrm{~Hz}, 1 \mathrm{H}), 6.60-6.62(\mathrm{~m}, 1 \mathrm{H}), 7.05-7.07(\mathrm{~m}, 1 \mathrm{H}), 7.27-7.31(\mathrm{~m}, 1 \mathrm{H})$, 7.33$7.39(\mathrm{~m}, 8 \mathrm{H}), 7.56-7.57(\mathrm{~m}, 2 \mathrm{H}) ;{ }^{13} \mathrm{C} \mathrm{NMR}\left(125 \mathrm{MHz}, \mathrm{CDCl}_{3}\right)$ \&: 59.4, 67.2, 74.0, 78.5, 79.7, 83.0, 106.5, 111.3, 117.0, 121.8, 126.6 (2C), 127.7, 128.4 (2C), 128.8 (2C), 129.1, 130.7, 132.4 (2C), 133.4, 139.4, 149.4; HRMS (FAB) calcd for $\mathrm{C}_{24} \mathrm{H}_{20} \mathrm{NO}\left(\mathrm{MH}^{+}\right) 338.1539$, found 338.1537.

$( \pm)-(1 R, 2 S)-2-[(2-B r o m o p h e n y l) a m i n o]-1,2-d i p h e n y l e t h a n-1-o l ~[( \pm)-S 5]$. The reaction of 2bromoiodobenzene and $( \pm)$-S4 was carried out according to the reported method ${ }^{14}$ as follows: a mixture of 2-bromoiodobenzene $(0.9 \mathrm{~mL}, 7.01 \mathrm{mmol}),( \pm)-\mathrm{S} 4(1.72 \mathrm{~g}, 8.06 \mathrm{mmol}), \mathrm{NaOH}(600 \mathrm{mg}$, $15.0 \mathrm{mmol})$, and $\mathrm{CuI}(35.7 \mathrm{mg}, 0.19 \mathrm{mmol})$ was stirred under argon at $90{ }^{\circ} \mathrm{C}$ for $13 \mathrm{~h}$. The reaction mixture was diluted with EtOAc, washed water and brine, dried over $\mathrm{Na}_{2} \mathrm{SO}_{4}$, and concentrated in vacuo. The residue was chromatographed on silica gel (hexane/EtOAc $=10 / 1)$ to afford $( \pm)$-S5 (2.06 g, 80\%) as pale amber powder: $\mathrm{mp} 100{ }^{\circ} \mathrm{C}$; IR (neat): $3398(\mathrm{OH}), 1321(\mathrm{NH})$; ${ }^{1} \mathrm{H}$ NMR $(500$ $\mathrm{MHz}_{\mathrm{CDCl}}$ ) $\delta:$ 2.37-2.39 (br m, 1H), 4.67-4.69 (m, 1H), 5.06-5.07 (m, 1H), 5.14-5.16 (br m, 1H), $6.35(\mathrm{~d}, J=8.0 \mathrm{~Hz}, 1 \mathrm{H}), 6.48(\mathrm{t}, J=7.7 \mathrm{~Hz}, 1 \mathrm{H}), 6.93(\mathrm{t}, J=7.7 \mathrm{~Hz}, 1 \mathrm{H}), 7.11-7.12(\mathrm{~m}, 4 \mathrm{H}), 7.23-$

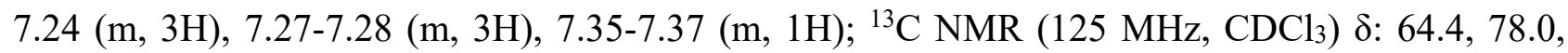
$110.5,112.8,118.0,126.5(2 \mathrm{C}), 127.1(2 \mathrm{C}), 127.7,128.0,128.2,128.3(2 \mathrm{C}), 128.6(2 \mathrm{C}), 132.2$, 139.6, 140.4, 143.9. Anal. calcd for $\mathrm{C}_{20} \mathrm{H}_{18}$ BrNO: C, 65.23; H, 4.93; N, 3.80. Found: C, 65.48; H, $4.89 ; \mathrm{N}, 3.79$.

\section{$( \pm)-(1 R, 2 S)-1,2-D i p h e n y l-2-(\{2-[($ trimethylsilyl)ethynyl]phenyl\}amino)ethan-1-ol $[( \pm)-S 6]$.}

According to the procedure described for the preparation of S2, $( \pm)-\mathbf{S 5}(2.95 \mathrm{~g}, 8.01 \mathrm{mmol})$ was 
converted to $( \pm)$-S6 (2.72 g, 88\%). Column chromatography: silica gel (hexane/EtOAc $=10 / 1)$ : dark brown oil; IR (neat): $3298(\mathrm{OH}), 2140(\mathrm{C} \equiv \mathrm{C}), 1252(\mathrm{NH}) ;{ }^{1} \mathrm{H}$ NMR $\left(500 \mathrm{MHz}, \mathrm{CDCl}_{3}\right) \delta: 0.31$ (s, 9H), 2.30-2.31 (br m, 1H), 4.73-4.74 (br m, 1H), 5.10-5.11 (br m, 1H), 5.57-5.58 (br m, 1H), 6.36 $(\mathrm{d}, J=8.0 \mathrm{~Hz}, 1 \mathrm{H}), 6.53-6.54(\mathrm{~m}, 1 \mathrm{H}), 6.97-6.99(\mathrm{~m}, 1 \mathrm{H}), 7.09-7.11(\mathrm{~m}, 4 \mathrm{H}), 7.22-7.27(\mathrm{~m}, 7 \mathrm{H})$; ${ }^{13} \mathrm{C}$ NMR (125 MHz, $\left.\mathrm{CDCl}_{3}\right) \delta: 0.0$ (3C), 63.2, 76.9, 100.2, 101.7, 108.0, 110.8, 116.5, 126.3 (2C), 127.4, 127.6 (2C), 127.7, 127.96 (2C), 128.02 (2C), 129.7, 132.0, 137.9, 139.7, 147.8; HRMS (FAB) calcd for $\mathrm{C}_{25} \mathrm{H}_{28} \mathrm{NOSi}\left(\mathrm{MH}^{+}\right)$386.1935, found 386.1927 .

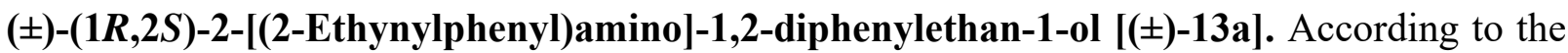
procedure described for the preparation of S3, $( \pm)-\mathbf{S 6}(1.44 \mathrm{~g}, 3.74 \mathrm{mmol})$ was converted into $( \pm)$ 13a ( $852 \mathrm{mg}, 73 \%$ ). Column chromatography: silica gel (hexane/ $\mathrm{CHCl}_{3}=$ from $1 / 1$ to $1 / 2$ ): amber oil; IR (neat): $3396(\mathrm{OH}), 3292(\mathrm{C} \equiv \mathrm{CH}), 2094(\mathrm{C} \equiv \mathrm{C}) ;{ }^{1} \mathrm{H}$ NMR (500 MHz, $\left.\mathrm{CDCl}_{3}\right)$ 8: 2.54-2.55 (br $\mathrm{m}, 1 \mathrm{H}), 3.38(\mathrm{~s}, 1 \mathrm{H}), 4.70-4.71$ (br m, 1H), 5.02-5.03 (br m, 1H), 5.44-5.45 (br m, 1H), $6.30(\mathrm{~d}, J=$ 8.6 Hz, 1H), 6.52-6.53 (m, 1H), 6.94-6.98 (m, 1H), 7.06-7.08 (m, 4H), 7.17-7.28 (m, 7H); ${ }^{13} \mathrm{C} \mathrm{NMR}$ $\left(125 \mathrm{MHz} \mathrm{CDCl}_{3}\right) \delta: 63.1,77.2,80.6,83.1,107.0,111.1,116.7,126.6(2 \mathrm{C}), 127.55,127.63$ (2C), 128.0, 128.1 (2C), 128.2 (2C), 130.1, 132.4, 138.3, 139.5, 148.2; HRMS (FAB) calcd for $\mathrm{C}_{22} \mathrm{H}_{20} \mathrm{NO}$ $\left(\mathrm{MH}^{+}\right)$314.1539, found 314.1535.

$( \pm)-(1 R, 2 S)-1,2-D i p h e n y l-2-\{[2-(p h e n y l b u t a-1,3-d i y n-1-y l) p h e n y l]$ amino $\}$ ethan-1-ol $[( \pm)-$ $\mathbf{2 b}$. According to the procedure described for the preparation of 2a, $( \pm)-\mathbf{1 3 a}(430 \mathrm{mg}, 1.37 \mathrm{mmol})$ was converted into $( \pm)-\mathbf{2 b}(337 \mathrm{mg}, 59 \%)$. Column chromatography: silica gel (hexane $/ \mathrm{CHCl}_{3}=1 / 1$ to $\mathrm{CHCl}_{3}$ only): brown powder; mp $122-124{ }^{\circ} \mathrm{C}$; IR (neat): $3401(\mathrm{OH}), 2209(\mathrm{C} \equiv \mathrm{C}), 2141(\mathrm{C} \equiv \mathrm{C})$; ${ }^{1} \mathrm{H}$ NMR (500 MHz, $\mathrm{CDCl}_{3}$ ) $\delta:$ 2.43-2.43 (br m, 1H), 4.72-4.73 (br m, 1H), 5.05-5.06 (br m, 1H), 5.45-5.46 (br m, 1H), $6.29(\mathrm{~d}, J=8.6 \mathrm{~Hz}, 1 \mathrm{H}), 6.54(\mathrm{t}, J=7.4 \mathrm{~Hz}, 1 \mathrm{H}), 6.98(\mathrm{t}, J=7.4 \mathrm{~Hz}, 1 \mathrm{H})$, $7.16(\mathrm{~d}, J=6.9 \mathrm{~Hz}, 4 \mathrm{H}), 7.26-7.30(\mathrm{~m}, 7 \mathrm{H}), 7.36-7.41(\mathrm{~m}, 3 \mathrm{H}), 7.59-7.60(\mathrm{~m}, 2 \mathrm{H}) ;{ }^{13} \mathrm{C} \mathrm{NMR}(125$ $\left.\mathrm{MHz}, \mathrm{CDCl}_{3}\right) \delta: 63.4,74.1,77.5,78.5,79.9,82.9,106.6,111.2,116.9,122.0,126.6(2 \mathrm{C}), 127.6$ (2C), 127.8, 128.2, 128.4 (2C), 128.5 (2C), 128.6 (2C), 129.2, 130.7, 132.4 (2C), 132.9, 138.5, 139.2, 149.3; HRMS (FAB) calcd for $\mathrm{C}_{30} \mathrm{H}_{24} \mathrm{NO}\left(\mathrm{MH}^{+}\right)$414.1852, found 414.1860. 
Ethyl 2-Phenyl-2-(\{2-[(trimethylsilyl)ethynyl]phenyl\}amino)acetate [( \pm )-S8]. To a stirred suspension of $( \pm)-\mathrm{S} 7(2.59 \mathrm{~g}, 6.78 \mathrm{mmol}), \mathrm{PdCl}_{2}\left(\mathrm{PPh}_{3}\right)_{2}(119 \mathrm{mg}, 0.17 \mathrm{mmol})$ and $\mathrm{CuI}(32.3 \mathrm{mg}$, $0.17 \mathrm{mmol})$ in THF $(14 \mathrm{~mL})$ under argon was added trimethylsilylacetylene $(1.0 \mathrm{~mL}, 7.46 \mathrm{mmol})$ and $\mathrm{Et}_{3} \mathrm{~N}(4.3 \mathrm{~mL}, 33.9 \mathrm{mmol})$. After stirring at $\mathrm{rt}$ for $3 \mathrm{~h}$, the reaction mixture was diluted with EtOAc and filtered through a short pad of silica gel. The filtrate was concentrated in vacuo and the residue was chromatographed on silica gel (hexane/ $\left.\mathrm{CHCl}_{3}=3 / 1\right)$ to afford $( \pm)-\mathbf{S 8}(2.10 \mathrm{~g}, 88 \%)$ as amber oil: IR (neat): $2145(\mathrm{C} \equiv \mathrm{C}), 1736(\mathrm{C}=\mathrm{O}) ;{ }^{1} \mathrm{H}$ NMR $\left(500 \mathrm{MHz}, \mathrm{CDCl}_{3}\right) \delta: 0.31(\mathrm{~s}, 9 \mathrm{H}), 1.21(\mathrm{t}$, $J=7.2 \mathrm{~Hz}, 3 \mathrm{H}), 4.13-4.16(\mathrm{~m}, 1 \mathrm{H}), 4.23-4.26(\mathrm{~m}, 1 \mathrm{H}), 5.09(\mathrm{~d}, J=5.7 \mathrm{~Hz}, 1 \mathrm{H}), 6.04-6.05$ (br m, 1H), $6.29(\mathrm{~d}, J=8.6 \mathrm{~Hz}, 1 \mathrm{H}), 6.58-6.59(\mathrm{~m}, 1 \mathrm{H}), 7.02-7.03(\mathrm{~m}, 1 \mathrm{H}), 7.30(\mathrm{~d}, J=6.9 \mathrm{~Hz}, 2 \mathrm{H}), 7.34-$

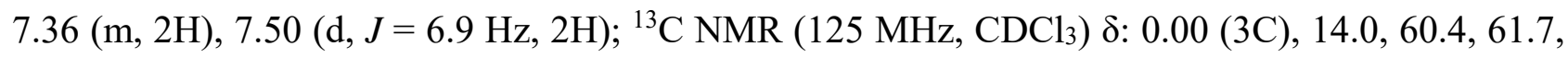
$100.8,108.1,101.5,110.3,116.8,127.0$ (2C), 128.2, 128.7 (2C), 129.8, 131.8, 137.4, 147.2, 171.0; HRMS (FAB) calcd for $\mathrm{C}_{21} \mathrm{H}_{26} \mathrm{NO}_{2} \mathrm{Si}\left(\mathrm{MH}^{+}\right)$352.1733, found 352.1726.

Ethyl 2-[(2-Ethynylphenyl)amino]-2-phenylacetate $[( \pm)-13 b]$. According to the procedure described for the preparation of S3, $( \pm)-\mathbf{S 8}(5.27 \mathrm{~g}, 15.0 \mathrm{mmol})$ was converted into $( \pm)-\mathbf{1 3 b}(2.99 \mathrm{~g}$, 71\%). Column chromatography: silica gel (hexane/EtOAc $=20 / 1)$ The product was recrystallized from $\mathrm{CHCl}_{3}$-hexane: white powder; mp $100{ }^{\circ} \mathrm{C}$; IR (neat): $3264(\mathrm{C} \equiv \mathrm{CH}), 2095(\mathrm{C} \equiv \mathrm{C}), 1723$ $(\mathrm{C}=\mathrm{O}) ;{ }^{1} \mathrm{H}$ NMR $\left(500 \mathrm{MHz}, \mathrm{CDCl}_{3}\right) \delta: 1.22(\mathrm{t}, J=7.2 \mathrm{~Hz}, 3 \mathrm{H}), 3.51(\mathrm{~s}, 1 \mathrm{H}), 4.15(\mathrm{dq}, J=11.0,7.0$ $\mathrm{Hz}, 1 \mathrm{H}), 4.24(\mathrm{dq}, J=11.0,7.0 \mathrm{~Hz}, 1 \mathrm{H}), 5.11(\mathrm{~d}, J=5.7 \mathrm{~Hz}, 1 \mathrm{H}), 6.03-6.04(\mathrm{br} \mathrm{m}, 1 \mathrm{H}), 6.30(\mathrm{~d}, J$ $=8.6 \mathrm{~Hz}, 1 \mathrm{H}), 6.61(\mathrm{ddd}, J=7.4,7.4,1.1 \mathrm{~Hz}, 1 \mathrm{H}), 7.05-7.07(\mathrm{~m}, 1 \mathrm{H}), 7.29-7.31(\mathrm{~m}, 1 \mathrm{H}), 7.35-7.37$ (m, 3H), 7.49-7.51 (m, 2H); ${ }^{13} \mathrm{C}$ NMR (125 MHz, $\left.\mathrm{CDCl}_{3}\right) \delta: 14.0,60.3,61.9,80.3,83.2,107.1$, $110.5,117.0,127.1$ (2C), 128.3, 128.8 (2C), 130.1, 132.6, 137.3, 147.3, 171.2. Anal. calcd for $\mathrm{C}_{18} \mathrm{H}_{17} \mathrm{NO}_{2}$ : C, 77.40; H, 6.13; N, 5.01. Found: C, 77.36; H, 6.11; N, 5.00.

Ethyl 2-\{[2-(Hepta-1,3-diyn-1-yl)phenyl]amino\}-2-phenylacetate [( \pm -S9]. According to the procedure described for the preparation of $\mathbf{2 a},( \pm)-\mathbf{1 3 b}(836 \mathrm{mg}, 3.0 \mathrm{mmol})$ was converted into $\mathbf{S 9}$ (621 mg, 60\%). Column chromatography: silica gel (hexane/EtOAc $=10 / 1)$ : brown powder; mp 83-84 ${ }^{\circ} \mathrm{C}$; IR (neat): $2236(\mathrm{C} \equiv \mathrm{C}), 2139(\mathrm{C} \equiv \mathrm{C}), 1733(\mathrm{C}=\mathrm{O}) ;{ }^{1} \mathrm{H}$ NMR $\left(500 \mathrm{MHz}, \mathrm{CDCl}_{3}\right) \delta: 1.04$ (t, 
$J=7.4 \mathrm{~Hz}, 3 \mathrm{H}), 1.23(\mathrm{t}, J=7.2 \mathrm{~Hz}, 3 \mathrm{H}), 1.63(\mathrm{qt}, J=7.4,7.2 \mathrm{~Hz}, 2 \mathrm{H}), 2.37(\mathrm{t}, J=7.2 \mathrm{~Hz}, 2 \mathrm{H})$, 4.14-4.27 (m, 2H), 5.09 (d, $J=6.3 \mathrm{~Hz}, 1 \mathrm{H}), 5.99-6.00$ (br m, 1H), 6.28 (d, J=8.0 Hz, 1H), 6.58$6.59(\mathrm{~m}, 1 \mathrm{H}), 7.02-7.04(\mathrm{~m}, 1 \mathrm{H}), 7.32-7.34(\mathrm{~m}, 4 \mathrm{H}), 7.50(\mathrm{~d}, J=7.4 \mathrm{~Hz}, 2 \mathrm{H}) ;{ }^{13} \mathrm{C} \mathrm{NMR}(125 \mathrm{MHz}$, $\left.\mathrm{CDCl}_{3}\right) \delta: 13.6,14.0,21.7,21.8,60.4,61.9,65.2,71.2,80.3,86.0,107.1,110.6,117.1,127.1(2 \mathrm{C})$, 128.3, 128.8 (2C), 130.2, 133.3, 137.2, 148.1, 171.0; HRMS (FAB) calcd for $\mathrm{C}_{23} \mathrm{H}_{24} \mathrm{NO}_{2}\left(\mathrm{MH}^{+}\right)$ 346.1802, found 346.1804.

2-\{[2-(Hepta-1,3-diyn-1-yl)phenyl]amino\}-2-phenylacetic acid $[( \pm)-2 c]$. To a stirred suspension of $( \pm)$-S9 $(86.9 \mathrm{mg}, 0.25 \mathrm{mmol})$ in EtOH $(5 \mathrm{~mL})$ was added THF until $( \pm)$-S9 dissolved (ca. $2 \mathrm{~mL}$ ), and $0.4 \mathrm{~N} \mathrm{NaOH}$ aq. $(19 \mathrm{~mL})$ was added to the reaction mixture. After stirring at $\mathrm{rt}$ for 40 min, the reaction mixture was diluted with $\mathrm{CH}_{2} \mathrm{Cl}_{2}$, washed with water, $1 \mathrm{~N} \mathrm{HCl}$ aq., and brine, dried over $\mathrm{Na}_{2} \mathrm{SO}_{4}$, and concentrated in vacuo. The residue was recrystallized from $\mathrm{CH}_{2} \mathrm{Cl}_{2}-$ hexane to afford $( \pm)-2 \mathrm{c}(43.3 \mathrm{mg}, 54 \%)$ : white solid; mp 164-166 ${ }^{\circ} \mathrm{C}$; IR (neat): $3394(\mathrm{OH}), 2238(\mathrm{C} \equiv \mathrm{C})$, $2147(\mathrm{C} \equiv \mathrm{C}), 1709(\mathrm{C}=\mathrm{O}) ;{ }^{1} \mathrm{H} \mathrm{NMR}\left(500 \mathrm{MHz}, \mathrm{CDCl}_{3}\right) \delta: 1.04(\mathrm{t}, J=7.4 \mathrm{~Hz}, 3 \mathrm{H}), 1.63(\mathrm{qt}, J=7.4$, $6.9 \mathrm{~Hz}, 2 \mathrm{H}), 2.37(\mathrm{t}, J=6.9 \mathrm{~Hz}, 2 \mathrm{H}), 5.14(\mathrm{~s}, 1 \mathrm{H}), 6.30-6.32(\mathrm{~m}, 1 \mathrm{H}), 6.61-6.63(\mathrm{~m}, 1 \mathrm{H})$, 7.04-7.08 (m, 1H), 7.35-7.37 (m, 4H), 7.51-7.52 (m, 2H); $\left.{ }^{13} \mathrm{C} \mathrm{NMR} \mathrm{(125} \mathrm{MHz,} \mathrm{CDCl}_{3}\right) \delta: 13.5,21.68,21.74$, $60.2,65.1,71.0,80.4,86.2,107.3,110.6,117.6,127.2(2 \mathrm{C}), 128.8,129.1(2 \mathrm{C}), 130.3,133.4,136.4$, 147.8, 175.8; HRMS (FAB) calcd for $\mathrm{C}_{21} \mathrm{H}_{20} \mathrm{NO}_{2}\left(\mathrm{MH}^{+}\right)$318.1489, found 318.1485.

$N$-(But-2-yn-1-yl)-N-(but-3-yn-1-yl)-4-methylbenzenesulfonamide (19). A mixture of 17 (4.47 g, $20.0 \mathrm{mmol})$ and $\mathrm{Cs}_{2} \mathrm{CO}_{3}(16.3 \mathrm{~g}, 50.0 \mathrm{mmol})$ in dry DMF (100 mL) was stirred in open atmospheric air at $0{ }^{\circ} \mathrm{C}$. After stirring at the same temperature for $0.5 \mathrm{~h}, 1$-bromobut-2-yne (18) (2.7 $\mathrm{mL}, 29.8 \mathrm{mmol}$ ) was added to the mixture. The mixture was stirred for $0.5 \mathrm{~h}$. The mixture was diluted with $\mathrm{Et}_{2} \mathrm{O}$, washed with water and brine, dried over $\mathrm{MgSO}_{4}$, and concentrated in vacuo. The residue was chromatographed on silica gel (hexane/EtOAc $=5 / 1)$ to afford $19(5.33 \mathrm{~g}, 97 \%)$ as colorless oil: IR (neat): $3288(\mathrm{C} \equiv \mathrm{CH}), 2224(\mathrm{C} \equiv \mathrm{C}), 2120(\mathrm{C} \equiv \mathrm{C}), 1343(\mathrm{~S}=\mathrm{O}), 1156(\mathrm{~S}=\mathrm{O}) ;{ }^{1} \mathrm{H}$ NMR (500 MHz, $\left.\mathrm{CDCl}_{3}\right) \delta: 1.58(\mathrm{t}, J=2.1 \mathrm{~Hz}, 3 \mathrm{H}), 2.01(\mathrm{t}, J=2.5 \mathrm{~Hz}, 1 \mathrm{H}), 2.42(\mathrm{~s}, 3 \mathrm{H}), 2.51(\mathrm{td}$, $J=7.4,2.5 \mathrm{~Hz}, 2 \mathrm{H}), 3.35(\mathrm{t}, J=7.4 \mathrm{~Hz}, 2 \mathrm{H}), 4.12(\mathrm{q}, J=2.1 \mathrm{~Hz}, 2 \mathrm{H}), 7.30(\mathrm{~d}, J=8.0 \mathrm{~Hz}, 2 \mathrm{H})$, 
$7.73(\mathrm{~d}, J=8.0 \mathrm{~Hz}, 2 \mathrm{H}) ;{ }^{13} \mathrm{C} \mathrm{NMR}\left(125 \mathrm{MHz}, \mathrm{CDCl}_{3}\right) \delta: 3.2,18.9,21.4,37.7,45.3,70.1,71.7,80.8$, 81.7, 127.6 (2C), 129.3 (2C), 135.9, 143.3; HRMS (FAB) calcd for $\mathrm{C}_{15} \mathrm{H}_{18} \mathrm{NO}_{2} \mathrm{~S}\left(\mathrm{MH}^{+}\right)$276.1058, found 276.1059 .

$N-(B u t-2-y n-1-y l)-N-[6-(2-\{[(1 R, 2 S)-2-h y d r o x y-1,2-d i p h e n y l e t h y l] a m i n o\}$ phenyl)hexa-3,5diyn-1-yl]-4-methylbenzenesulfonamide $[( \pm)-10 a]$. A mixture of $\mathbf{1 9}(1.10 \mathrm{~g}, 4.0 \mathrm{mmol}), \mathrm{AgNO}_{3}$ (203 $\mathrm{mg}, 1.20 \mathrm{mmol})$, and NIS $(1.26 \mathrm{~g}, 5.60 \mathrm{mmol})$ in acetone $(100 \mathrm{~mL})$ was stirred in open atmospheric air at room temperature under dark. After stirring at the room temperature for $1 \mathrm{~h}$, the mixture was concentrated in vacuo. The residue was diluted with $\mathrm{CHCl}_{3}$, washed with water and brine, dried over $\mathrm{MgSO}_{4}$, and concentrated in vacuo. This crude iodide 12 was used for the next reaction without further purification. According to the reported method, ${ }^{22}$ the copper-mediated coupling of $( \pm)$-13a and 12 was conducted as follows: 12, $( \pm)-\mathbf{1 3 a}(0.63 \mathrm{~g}, 2.02 \mathrm{mmol})$, and $\mathrm{CuCl}$ $(60.0 \mathrm{mg}, 0.60 \mathrm{mmol})$ in piperidine $(7.0 \mathrm{~mL})$ was stirred at room temperature under argon for $3 \mathrm{~h}$. The reaction mixture was quenched with aqueous saturated $\mathrm{NH}_{4} \mathrm{Cl}$, diluted with $\mathrm{Et}_{2} \mathrm{O}$, washed with brine, dried over $\mathrm{Na}_{2} \mathrm{SO}_{4}$, and concentrated in vacuo. The residue was chromatographed on silica gel (hexane/EtOAc $=10 / 1)$ to afford $( \pm)$-10a $(0.31 \mathrm{~g}, 19 \%$ based on $( \pm)$-13a) as pale amber amorphous: IR (neat): $3396(\mathrm{OH}), 2230(\mathrm{C} \equiv \mathrm{C}), 2214(\mathrm{C} \equiv \mathrm{C}), 1327(\mathrm{~S}=\mathrm{O}), 1157(\mathrm{~S}=\mathrm{O}) ;{ }^{1} \mathrm{H}$ NMR $\left(500 \mathrm{MHz}, \mathrm{CDCl}_{3}\right) \delta: 1.58(\mathrm{t}, J=2.0 \mathrm{~Hz}, 3 \mathrm{H}), 2.40(\mathrm{~s}, 3 \mathrm{H}), 2.59$ (br s, $\left.1 \mathrm{H}\right), 2.77$ (t, $\left.J=7.4 \mathrm{~Hz}, 2 \mathrm{H}\right)$, 3.44 (t, $J=7.4 \mathrm{~Hz}, 2 \mathrm{H}), 4.14-4.15$ (br m, 2H), 4.69-4.70 (br m, 1H), 5.07 (br s, 1H), 5.48-5.49 (br m, 1H), $6.26(\mathrm{~d}, J=8.6 \mathrm{~Hz}, 1 \mathrm{H}), 6.51(\mathrm{t}, J=7.2 \mathrm{~Hz}, 1 \mathrm{H}), 6.94-6.97(\mathrm{~m}, 1 \mathrm{H}), 7.11-7.11(\mathrm{~m}, 4 \mathrm{H}), 7.24-$ $7.28(\mathrm{~m}, 9 \mathrm{H}), 7.75(\mathrm{~d}, J=8.0 \mathrm{~Hz}, 2 \mathrm{H}) ;{ }^{13} \mathrm{C} \mathrm{NMR}\left(125 \mathrm{MHz}, \mathrm{CDCl}_{3}\right) \delta: 3.3,20.3,21.4,37.9,45.2$, $63.1,67.0,71.7,72.5,77.2,79.8,81.8,81.9,106.3,111.1,116.7,126.5(2 \mathrm{C}), 127.55,127.58(2 \mathrm{C})$, 127.7 (2C), 128.0, 128.15 (2C), 128.22 (2C), 129.4 (2C), 130.4, 132.8, 135.6, 138.3, 139.3, 143.5, 149.3; HRMS (FAB) calcd for $\mathrm{C}_{37} \mathrm{H}_{35} \mathrm{~N}_{2} \mathrm{O}_{3} \mathrm{~S}\left(\mathrm{MH}^{+}\right)$587.2368, found 587.2363.

Ethyl 2-\{[2-(6-\{[N-(But-2-yn-1-yl)-4-methylphenyl]sulfonamide $\}$ hexa-1,3-diyn-1yl)phenyl]amino\}-2-phenylacetate $[( \pm)-\mathbf{S 1 0})]$. According to the procedure described for the preparation of $( \pm)-\mathbf{1 0 a},( \pm)-\mathbf{1 3 b}(12.0 \mathrm{~g}, 3.0 \mathrm{mmol})$ was converted to $( \pm)-\mathbf{S 1 0}(0.80 \mathrm{~g}, 73 \%)$ by the 
reaction with 12 in the presence of $\mathrm{CuCl}(59.4 \mathrm{mg}, 0.6 \mathrm{mmol})$ in piperidine $(7 \mathrm{~mL})$ at room temperature for $4 \mathrm{~h}$. Column chromatography: silica gel (hexane/EtOAc $=3 / 1$ ); yellow oil; IR (neat): $2226(\mathrm{C} \equiv \mathrm{C}), 2146(\mathrm{C} \equiv \mathrm{C}), 1735(\mathrm{C}=\mathrm{O}), 1328(\mathrm{~S}=\mathrm{O}), 1158(\mathrm{~S}=\mathrm{O}) ;{ }^{1} \mathrm{H}$ NMR $(500 \mathrm{MHz}$, $\left.\mathrm{CDCl}_{3}\right) \delta: 1.22(\mathrm{t}, J=7.2 \mathrm{~Hz}, 3 \mathrm{H}), 1.61(\mathrm{t}, J=2.3 \mathrm{~Hz}, 3 \mathrm{H}), 2.41(\mathrm{~s}, 3 \mathrm{H}), 2.71-2.74(\mathrm{~m}, 2 \mathrm{H}), 3.39-$ $3.42(\mathrm{~m}, 2 \mathrm{H}), 4.12-4.27(\mathrm{~m}, 4 \mathrm{H}), 5.09(\mathrm{~d}, J=5.7 \mathrm{~Hz}, 1 \mathrm{H}), 5.98(\mathrm{~d}, J=5.7 \mathrm{~Hz}, 1 \mathrm{H}), 6.28(\mathrm{~d}, J=8.6$ $\mathrm{Hz}, 1 \mathrm{H}), 6.57-6.60(\mathrm{~m}, 1 \mathrm{H}), 7.02-7.05(\mathrm{~m}, 1 \mathrm{H}), 7.30-7.35(\mathrm{~m}, 6 \mathrm{H}), 7.49(\mathrm{~d}, J=7.4 \mathrm{~Hz}, 2 \mathrm{H}), 7.75(\mathrm{~d}$, $J=8.6 \mathrm{~Hz}, 2 \mathrm{H}) ;{ }^{13} \mathrm{C} \mathrm{NMR}\left(125 \mathrm{MHz}, \mathrm{CDCl}_{3}\right) \delta: 3.3,13.9,20.3,21.4,38.0,45.2,60.3,61.9,66.8$, 71.8, 72.2, 79.8, 81.8, 81.9, 106.6, 110.6, 117.1, 127.0 (2C), 127.7 (2C), 128.3, 128.8 (2C), 129.4 (2C), 130.4, 133.3, 135.8, 137.1, 143.4, 148.2, 170.9; HRMS (FAB) calcd for $\mathrm{C}_{33} \mathrm{H}_{33} \mathrm{~N}_{2} \mathrm{O}_{4} \mathrm{~S}\left(\mathrm{MH}^{+}\right)$ 553.2161 , found 553.2155

\section{2-\{[2-(6-\{[N-(But-2-yn-1-yl)-4-methylphenyl]sulfonamide $\}$ hexa-1,3-diyn-1-}

yl)phenyl]amino\}-2-phenylacetic Acid $[( \pm)-10 b]$. THF (ca. $2 \mathrm{~mL})$ was added to the mixture $( \pm)$ S10 $(0.15 \mathrm{~g}, 0.27 \mathrm{mmol})$ and $0.4 \mathrm{~N} \mathrm{NaOH}(2 \mathrm{~mL})$ in EtOH $(4 \mathrm{~mL})$. After stirring at the room temperature for $0.5 \mathrm{~h}$, the reaction mixture was diluted with $\mathrm{CH}_{2} \mathrm{Cl}_{2}$, washed with water, $1 \mathrm{~N} \mathrm{HCl}$, and brine, dried over $\mathrm{Na}_{2} \mathrm{SO}_{4}$, and concentrated in vacuo to afford $( \pm)-\mathbf{1 0 b}(0.14 \mathrm{~g}, 95 \%)$ as brown powder; mp 64-65 ${ }^{\circ} \mathrm{C}$; IR (neat): $3386(\mathrm{OH}), 2309(\mathrm{C} \equiv \mathrm{C}), 2145(\mathrm{C} \equiv \mathrm{C}), 1715(\mathrm{C}=\mathrm{O}), 1326(\mathrm{~S}=\mathrm{O})$, $1157(\mathrm{~S}=\mathrm{O}) ;{ }^{1} \mathrm{H}$ NMR $\left(500 \mathrm{MHz}, \mathrm{CDCl}_{3}\right) \delta: 1.60$ (br s, 3H), 2.42 (s, 3H), 2.71-2.72 (br m, 2H), 3.39-3.41 (br m, 2H), 4.12 (br s, 2H), 5.12 (br s, 1H), 6.31-6.32 (br m, 1H), 6.61-6.63 (m, 1H), 7.06-7.07 (m, 1H), 7.29-7.37 (m, 7H), 7.50-7.52 (m, 2H), $7.75(\mathrm{~d}, J=8.0 \mathrm{~Hz}, 2 \mathrm{H}) ;{ }^{13} \mathrm{C}$ NMR $(125$ $\left.\mathrm{MHz}, \mathrm{CDCl}_{3}\right) \delta: 3.3,20.3,21.5,37.9,45.1,60.2,66.8,71.8,72.2,79.8,81.9,82.1,106.6,110.7$, 117.4, 127.2 (2C), 127.7 (2C), 128.6, 129.0 (2C), 129.4 (2C), 130.5, 133.5, 135.7, 136.5, 143.5, 148.0, 176.0; HRMS (FAB) calcd for $\mathrm{C}_{31} \mathrm{H}_{29} \mathrm{~N}_{2} \mathrm{O}_{4} \mathrm{~S}\left(\mathrm{MH}^{+}\right)$525.1848, found 525.1849.

Ethyl 4-\{[N-(But-2-yn-1-yl)-4-methylphenyl]sulfonamide\}butanoate (23). The coupling of 21 and ethyl 4-bromobutanoate (22) was carried out according to the reported method ${ }^{23}$ as follows: a mixture of 21 (448 mg, $2.0 \mathrm{mmol})$ and $\mathrm{NaH}(48.0 \mathrm{mg}, 2.4 \mathrm{mmol})$ in dry DMF (5 mL) was stirred at room temperature for $0.5 \mathrm{~h}$ under argon. 4-Bromobutanoate $\mathbf{( 2 2})(0.17 \mathrm{~mL}, 2.4 \mathrm{mmol})$ was added 
to the reaction mixture. The mixture was stirred for $3 \mathrm{~h}$. The mixture was quenched with aqueous saturated $\mathrm{NH}_{4} \mathrm{Cl}$, diluted with EtOAc, washed with brine, dried over $\mathrm{Na}_{2} \mathrm{SO}_{4}$, and concentrated in vacuo. The residue was chromatographed on silica gel (hexane/EtOAc $=10 / 1)$ to afford $23(0.68 \mathrm{~g}$, 100\%) as pale yellow oil: IR (neat): $1730(\mathrm{C}=\mathrm{O}), 1345(\mathrm{~S}=\mathrm{O}), 1157(\mathrm{~S}=\mathrm{O}) ;{ }^{1} \mathrm{H}$ NMR $(500 \mathrm{MHz}$, $\left.\mathrm{CDCl}_{3}\right) \delta: 1.26(\mathrm{t}, J=7.2 \mathrm{~Hz}, 3 \mathrm{H}), 1.54(\mathrm{t}, J=2.3 \mathrm{~Hz}, 3 \mathrm{H}), 1.88(\mathrm{tt}, J=6.6,6.6 \mathrm{~Hz}, 2 \mathrm{H}), 2.40-2.41$ $(\mathrm{m}, 5 \mathrm{H}), 3.21(\mathrm{t}, J=6.6 \mathrm{~Hz}, 2 \mathrm{H}), 4.05(\mathrm{q}, J=2.3 \mathrm{~Hz}, 2 \mathrm{H}), 4.14(\mathrm{q}, J=7.2 \mathrm{~Hz}, 2 \mathrm{H}), 7.29(\mathrm{~d}, J=8.0$ $\mathrm{Hz}, 2 \mathrm{H}), 7.72(\mathrm{~d}, J=8.0 \mathrm{~Hz}, 2 \mathrm{H}) ;{ }^{13} \mathrm{C} \mathrm{NMR}\left(125 \mathrm{MHz}, \mathrm{CDCl}_{3}\right) \delta: 3.2,14.2,21.5,22.7,31.1,36.8$, 45.5, 60.5, 71.6, 81.6, 127.8 (2C), 129.2 (2C), 136.0, 143.2, 173.1; HRMS (FAB) calcd for $\mathrm{C}_{17} \mathrm{H}_{24} \mathrm{NO}_{4} \mathrm{~S}\left(\mathrm{MH}^{+}\right)$338.1426, found 338.1423.

\section{N-(But-2-yn-1-yl)-N-[4-hydroxy-6-(trimethylsilyl)hex-5-yn-1-yl]-4-}

methylbenzenesulfonamide (24). Alkynylation was carried out according to the reported method ${ }^{24}$ as follows: to a mixture of $\mathbf{2 3}(2.70 \mathrm{~g}, 8.0 \mathrm{mmol})$ in dry $\mathrm{CH}_{2} \mathrm{Cl}_{2}(40 \mathrm{~mL})$ was added $1 \mathrm{M}$ DIBAL in toluene $(9 \mathrm{~mL}, 8.7 \mathrm{mmol})$ at $-78{ }^{\circ} \mathrm{C}$ under argon. After stirring at the same temperature for $1 \mathrm{~h}$, the reaction was quenched with $\mathrm{MeOH}$ ( 1 equiv) and $\mathrm{H}_{2} \mathrm{O}$ (6 equiv) at $-78^{\circ} \mathrm{C}$, and the resulting slurry was allowed to warm to room temperature. It was then filtered through $\mathrm{MgSO}_{4}$ and Celite and the solvent was evaporated under reduced pressure to leave aldehyde $\mathbf{1 4}$ as a yellow liquid. This crude material was used for the next reaction without further purification. To a mixture of trimethylsilylacetylene $(1 \mathrm{~mL}, 7.20 \mathrm{mmol})$ in dry THF $(36 \mathrm{~mL})$ at $-78{ }^{\circ} \mathrm{C}$ under argon was added $n$ BuLi in THF (2.6M solution in $n$-BuLi; $3.1 \mathrm{~mL}, 8.0 \mathrm{mmol})$ dropwise, and the mixture was stirred at $-78^{\circ} \mathrm{C}$ for $0.5 \mathrm{~h}$ to afford a solution of lithium trimethylsilylacetylide (15), to which the solution of 14 in THF (18 mL) was slowly added. After stirring at $-78{ }^{\circ} \mathrm{C}$ for $2 \mathrm{~h}$, the reaction mixture was cooled to room temperature, quenched with aqueous saturated $\mathrm{NH}_{4} \mathrm{Cl}$, diluted with $\mathrm{Et}_{2} \mathrm{O}$, washed with brine, dried over $\mathrm{MgSO}_{4}$, and concentrated in vacuo. The residue was chromatographed on silica gel (hexane/EtOAc =3/1) to afford $24(2.81 \mathrm{~g}, 90 \%)$ as colorless oil: IR (neat): $3511(\mathrm{OH})$, $2223(\mathrm{C} \equiv \mathrm{C}), 2170(\mathrm{C} \equiv \mathrm{C}), 1345(\mathrm{~S}=\mathrm{O}), 1158(\mathrm{~S}=\mathrm{O}) ;{ }^{1} \mathrm{H}$ NMR $\left(500 \mathrm{MHz}, \mathrm{CDCl}_{3}\right) \delta: 0.17(\mathrm{~s}, 9 \mathrm{H})$, $1.53(\mathrm{t}, J=2.3 \mathrm{~Hz}, 3 \mathrm{H}), 1.72-1.77(\mathrm{~m}, 4 \mathrm{H}), 2.03-2.04($ br m, 1H), $2.42(\mathrm{~s}, 3 \mathrm{H}), 3.20-3.21$ (br m, 2H), 
$4.06(\mathrm{q}, J=2.3 \mathrm{~Hz}, 2 \mathrm{H}), 4.42-4.43($ br m, $1 \mathrm{H}), 7.28-7.30(\mathrm{~m}, 2 \mathrm{H}), 7.72-7.73(\mathrm{~m}, 2 \mathrm{H}) ;{ }^{13} \mathrm{C} \mathrm{NMR}$ (125 MHz, $\left.\mathrm{CDCl}_{3}\right) \delta: 0.0$ (3C), 3.4, 21.7, 23.1, 34.4, 36.6, 45.8, 62.5, 71.7, 81.7, 89.9, 106.4, 128.0 (2C), 129.4 (2C), 136.1, 143.3; HRMS (FAB) calcd for $\mathrm{C}_{20} \mathrm{H}_{30} \mathrm{NO}_{3} \mathrm{SSi}\left(\mathrm{MH}^{+}\right)$392.1716, found 392.1710.

$N$-(But-2-yn-1-yl)-N-(4-hydroxyhex-5-yn-1-yl)-4-methylbenzenesulfonamide (25). To a mixture of $24(4.48 \mathrm{~g}, 11.4 \mathrm{mmol})$ in dry THF $(23 \mathrm{~mL})$ at $0{ }^{\circ} \mathrm{C}$ under argon was added $1 \mathrm{M}$ TBAF in THF (11.5 mL, $11.4 \mathrm{mmol})$ dropwise, and the mixture was stirred at room temperature for $0.7 \mathrm{~h}$. The mixture was diluted with $\mathrm{CH}_{2} \mathrm{Cl}_{2}$, washed with water and brine, dried over $\mathrm{Na}_{2} \mathrm{SO}_{4}$, and concentrated in vacuo. The residue was chromatographed on silica gel (hexane/EtOAc $=5 / 1$ ) to afford 25 (3.42 g, 94\%) as pale amber oil: IR (neat): $3516(\mathrm{OH}), 3284(\mathrm{C} \equiv \mathrm{H}), 2225(\mathrm{C} \equiv \mathrm{C}), 2114$ $(\mathrm{C} \equiv \mathrm{C}), 1327(\mathrm{~S}=\mathrm{O}), 1156(\mathrm{~S}=\mathrm{O}) ;{ }^{1} \mathrm{H}$ NMR $\left(500 \mathrm{MHz}, \mathrm{CDCl}_{3}\right) \delta: 1.54$ (br s, 3H), 1.72-1.81 (m, 4H), 2.31 (br s, 1H), 2.42 (s, 3H), 2.48-2.48 (br m, 1H), 3.21 (t, $J=6.6 \mathrm{~Hz}, 2 \mathrm{H}), 4.06-4.06$ (br m, 2H), 4.45 (br s, $1 \mathrm{H}), 7.29(\mathrm{~d}, J=8.0 \mathrm{~Hz}, 2 \mathrm{H}), 7.72(\mathrm{~d}, J=8.0 \mathrm{~Hz}, 2 \mathrm{H}) ;{ }^{13} \mathrm{C} \mathrm{NMR}\left(125 \mathrm{MHz}, \mathrm{CDCl}_{3}\right) \delta$ : 3.2. $21.4,22.8,34.1,36.5,45.6,61.6,71.5,73.1,81.6,84.5,127.8(2 \mathrm{C}), 129.2(2 \mathrm{C}), 135.8,143.2$; HRMS (FAB) calcd for $\mathrm{C}_{17} \mathrm{H}_{22} \mathrm{NO}_{3} \mathrm{~S}\left(\mathrm{MH}^{+}\right) 320.1320$, found 320.1318 .

\section{$N$-[6-(2-Aminophenyl)-4-hydroxyhex-5-yn-1-yl]-N-(but-2-yn-1-yl)-4-}

methylbenzenesulfonamide (26). $\mathrm{Et}_{3} \mathrm{~N}(1.5 \mathrm{~mL}, 12.0 \mathrm{mmol})$ was added to a stirred mixture of 25 (0.97 g, $3.02 \mathrm{mmol})$, 2-iodoaniline (16) (0.66 g, $3.03 \mathrm{mmol}), \mathrm{PdCl}_{2}\left(\mathrm{PPh}_{3}\right) 2(53.1 \mathrm{mg}, 0.08 \mathrm{mmol})$ and $\mathrm{CuI}(28.8 \mathrm{mg}, 0.15 \mathrm{mmol})$ in $\mathrm{CH}_{3} \mathrm{CN}(20 \mathrm{~mL})$ under argon. After stirring at room temperature for $1.5 \mathrm{~h}$, the mixture was diluted with EtOAc, washed with aqueous saturated $\mathrm{NH}_{4} \mathrm{Cl}$ and brine, dried over $\mathrm{Na}_{2} \mathrm{SO}_{4}$, and concentrated in vacuo. The residue was chromatographed on silica gel (hexane/EtOAc $=2 / 1)$ to afford $26(1.11 \mathrm{~g}, 90 \%)$ as colorless oil: IR (neat): $3379(\mathrm{OH}), 2301(\mathrm{C} \equiv \mathrm{C})$, $2218(\mathrm{C} \equiv \mathrm{C}), 1328(\mathrm{~S}=\mathrm{O}), 1306(\mathrm{NH}), 1156(\mathrm{~S}=\mathrm{O}) ;{ }^{1} \mathrm{H}$ NMR $\left(500 \mathrm{MHz}, \mathrm{CDCl}_{3}\right) \delta: 1.52(\mathrm{t}, J=2.3$ $\mathrm{Hz}, 3 \mathrm{H}), 1.78-1.90(\mathrm{~m}, 4 \mathrm{H}), 2.40(\mathrm{~s}, 3 \mathrm{H}), 2.49(\mathrm{br} \mathrm{s}, 1 \mathrm{H}), 3.24(\mathrm{t}, J=6.6 \mathrm{~Hz}, 2 \mathrm{H}), 4.06(\mathrm{q}, J=2.3$ Hz, 2H), 4.24 (br s, 2H), 4.70-4.72 (br m, 1H), 6.65-6.68 (m, 2H), 7.09-7.13 (m, 1H), 7.23-7.27 (m, 3H), $7.72(\mathrm{~d}, J=8.0 \mathrm{~Hz}, 2 \mathrm{H}) ;{ }^{13} \mathrm{C} \mathrm{NMR}\left(125 \mathrm{MHz}, \mathrm{CDCl}_{3}\right) \delta: 3.2,21.4,23.2,34.6,36.7,45.8,62.5$, 
71.6, 81.6, 81.7, 95.1, 107.1, 114.3, 117.8, 127.8 (2C), 129.2 (2C), 129.8, 132.2, 135.8, 143.2, 147.9; HRMS (FAB) calcd for $\mathrm{C}_{23} \mathrm{H}_{27} \mathrm{~N}_{2} \mathrm{O}_{3} \mathrm{~S}\left(\mathrm{MH}^{+}\right)$411.1742, found 411.1742.

\section{$N$-[6-(2-Aminophenyl)-4-oxohex-5-yn-1-yl]-N-(but-2-yn-1-yl)-4-}

methylbenzenesulfonamide (27). According to the reported method, ${ }^{25}$ oxidation of 26 was conducted as follows: a mixture of $26(421 \mathrm{mg}, 1.03 \mathrm{mmol})$ and $\mathrm{MnO}_{2}(882 \mathrm{mg}, 10.3 \mathrm{mmol})$ in dry $\mathrm{CHCl}_{3}(10 \mathrm{~mL})$ was stirred under reflux for $0.5 \mathrm{~h}$. The reaction mixture was cooled to room temperature, filtered through Celite, and concentrated in vacuo. The residue was chromatographed on silica gel (hexane/EtOAc $=5 / 1)$ to afford $27(297 \mathrm{mg}, 71 \%)$ as orange amber oil: IR (neat): 2300 $(\mathrm{C} \equiv \mathrm{C}), 2180(\mathrm{C} \equiv \mathrm{C}), 1658(\mathrm{C}=\mathrm{O}), 1342(\mathrm{~S}=\mathrm{O}), 1330(\mathrm{NH}), 1156(\mathrm{~S}=\mathrm{O}) ;{ }^{1} \mathrm{H}$ NMR $(500 \mathrm{MHz}$, $\left.\mathrm{CDCl}_{3}\right) \delta: 1.54(\mathrm{t}, J=2.3 \mathrm{~Hz}, 3 \mathrm{H}), 1.99(\mathrm{tt}, J=7.0,7.0 \mathrm{~Hz}, 2 \mathrm{H}), 2.41(\mathrm{~s}, 3 \mathrm{H}), 2.82(\mathrm{t}, J=7.2 \mathrm{~Hz}$, 2H), $3.22(\mathrm{t}, J=6.6 \mathrm{~Hz}, 2 \mathrm{H}), 4.05(\mathrm{q}, J=2.3 \mathrm{~Hz}, 2 \mathrm{H}), 4.47(\mathrm{br} \mathrm{s}, 2 \mathrm{H}), 6.68-6.69(\mathrm{~m}, 2 \mathrm{H}), 7.20-7.24$ $(\mathrm{m}, 1 \mathrm{H}), 7.28(\mathrm{~d}, J=8.6 \mathrm{~Hz}, 2 \mathrm{H}), 7.36-7.37(\mathrm{~m}, 1 \mathrm{H}), 7.72(\mathrm{~d}, J=8.0 \mathrm{~Hz}, 2 \mathrm{H}) ;{ }^{13} \mathrm{C} \mathrm{NMR}(125 \mathrm{MHz}$, $\left.\mathrm{CDCl}_{3}\right) \delta: 3.2,21.4,21.9,37.0,42.1,45.5,71.5,81.7,89.1,94.1,103.4,114.5,117.8,127.8(2 \mathrm{C})$, 129.2 (2C), 132.5, 133.8, 135.7, 143.3, 105.3, 186.5; HRMS (FAB) calcd for $\mathrm{C}_{23} \mathrm{H}_{25} \mathrm{~N}_{2} \mathrm{O}_{3} \mathrm{~S}\left(\mathrm{MH}^{+}\right)$ 409.1586, found 409.1590.

(E)- and (Z)- $N$-\{6-(2-Aminophenyl)-4-[(triisopropylsilyl)oxy]hex-3-en-5-yn-1-yl\}- $N$-(but-2yn-1-yl)-4-methylbenzenesulfonamide (11a). TIPSOTf (0.9 mL, $3.24 \mathrm{mmol})$ was added dropwise to a mixture of $27(883 \mathrm{mg}, 2.16 \mathrm{mmol})$ and $\mathrm{Et}_{3} \mathrm{~N}(0.8 \mathrm{~mL}, 6.84 \mathrm{mmol})$ in dry $\mathrm{CH}_{2} \mathrm{Cl}_{2}(36 \mathrm{~mL})$ at $78^{\circ} \mathrm{C}$ under argon, and the mixture was stirred for $2 \mathrm{~h}$. The mixture allowed to warm slowly to room temperature. The mixture was diluted with EtOAc, washed with $3 \mathrm{~N} \mathrm{HCl}$ twice, water, aqueous saturated $\mathrm{NaHCO}_{3}$ and brine, dried over $\mathrm{Na}_{2} \mathrm{SO}_{4}$, and concentrated in vacuo. The residue was chromatographed on silica gel (hexane/EtOAc $=5 / 1)$ to afford 11a $(921 \mathrm{mg}, 75 \%, Z / E=85 / 15$, determined by ${ }^{1} \mathrm{H}$ NMR). ${ }^{11}$ Both products were isolated by column chromatography on silica gel followed by PTLC (silica gel) with hexane/EtOAc (10/1). Compound (Z)-11a (more polar isomer): amber oil; IR (neat): $2193(\mathrm{C} \equiv \mathrm{C}), 1616(\mathrm{SiOC}=\mathrm{C}) ;{ }^{1} \mathrm{H}$ NMR $\left(500 \mathrm{MHz}, \mathrm{CDCl}_{3}\right) \delta: 1.12(\mathrm{~d}, J=7.4$ $\mathrm{Hz}, 18 \mathrm{H}), 1.27-1.36(\mathrm{~m}, 3 \mathrm{H}), 1.54(\mathrm{t}, J=2.3 \mathrm{~Hz}, 3 \mathrm{H}), 2.41(\mathrm{~s}, 3 \mathrm{H}), 2.47-2.53(\mathrm{~m}, 2 \mathrm{H}), 3.22(\mathrm{t}, J=$ 
$7.4 \mathrm{~Hz}, 2 \mathrm{H}), 4.09$ (q, $J=2.3 \mathrm{~Hz}, 2 \mathrm{H}), 4.19(\mathrm{br} \mathrm{s}, 2 \mathrm{H}), 5.09$ (t, $J=7.2 \mathrm{~Hz}, 1 \mathrm{H}), 6.67-6.69$ (m, 2H), 7.11-7.14 (m, 1H), $7.21(\mathrm{~d}, J=6.9 \mathrm{~Hz}, 1 \mathrm{H}), 7.28(\mathrm{~d}, J=8.0 \mathrm{~Hz}, 2 \mathrm{H}), 7.74(\mathrm{~d}, J=8.0 \mathrm{~Hz}, 2 \mathrm{H}) ;{ }^{13} \mathrm{C}$ NMR (125 MHz, $\left.\mathrm{CDCl}_{3}\right) \delta:$ 3.2, 12.9 (3C), 18.0 (6C), 21.5, 24.4, 36.7, 45.3, 71.8, 81.4, 83.7, 92.3, 107.1, 114.3, 114.8, 117.8, 127.8 (2C), 129.2 (2C), 129.9, 131.9, 134.1, 136.1, 143.1, 148.0; HRMS (FAB) calcd for $\mathrm{C}_{32} \mathrm{H}_{45} \mathrm{~N}_{2} \mathrm{O}_{3} \mathrm{SSi}\left(\mathrm{MH}^{+}\right)$565.2920, found 565.2919. Compound (E)-11a (less polar isomer): amber oil; IR (neat): $2191(\mathrm{C} \equiv \mathrm{C}), 1616(\mathrm{SiOC}=\mathrm{C}) ;{ }^{1} \mathrm{H}$ NMR $\left(500 \mathrm{MHz}, \mathrm{CDCl}_{3}\right) \delta: 1.13(\mathrm{~d}$, $J=7.4 \mathrm{~Hz}, 18 \mathrm{H}), 1.27(\mathrm{~m}, 3 \mathrm{H}), 1.51(\mathrm{t}, J=2.3 \mathrm{~Hz}, 3 \mathrm{H}), 2.40(\mathrm{~s}, 3 \mathrm{H}), 2.51-2.54(\mathrm{~m}, 2 \mathrm{H}), 3.21(\mathrm{t}, J=$ $7.4 \mathrm{~Hz}, 2 \mathrm{H}), 4.10(\mathrm{q}, J=2.3 \mathrm{~Hz}, 2 \mathrm{H}), 4.27(\mathrm{br} \mathrm{s}, 2 \mathrm{H}), 5.30(\mathrm{t}, J=8.0 \mathrm{~Hz}, 1 \mathrm{H}), 6.67-6.70(\mathrm{~m}, 2 \mathrm{H})$, 7.11-7.14 (m, 1H), 7.26-7.27 (m, 3H), $7.72(\mathrm{~d}, J=8.0 \mathrm{~Hz}, 2 \mathrm{H}) ;{ }^{13} \mathrm{C} \mathrm{NMR}\left(125 \mathrm{MHz}, \mathrm{CDCl}_{3}\right) \delta: 3.2$, 12.5 (3C), 17.9 (6C), 21.5, 27.4, 37.0, 45.9, 71.8, 81.6, 89.1, 89.8, 107.0, 114.4, 114.6, 117.7, 127.8 (2C), 129.2 (2C), 130.0, 132.1, 135.8, 136.0, 143.1, 148.0; HRMS (FAB) calcd for $\mathrm{C}_{32} \mathrm{H}_{45} \mathrm{~N}_{2} \mathrm{O}_{3} \mathrm{SSi}$ $\left(\mathrm{MH}^{+}\right)$565.2920, found 565.2913.

(E)- and (Z)- $N$-\{6-(2-Aminophenyl)-4-[(tert-butyldimethylsilyl)oxy]hex-3-en-5-yn-1-yl\}-N(but-2-yn-1-yl)-4-methylbenzenesulfonamide (11b). TBSOTf $(0.2 \mathrm{~mL}, 0.81 \mathrm{mmol})$ was added dropwise to a mixture of $27(166 \mathrm{mg}, 0.41 \mathrm{mmol})$ and $\mathrm{Et}_{3} \mathrm{~N}(0.1 \mathrm{~mL}, 0.81 \mathrm{mmol})$ in dry $\mathrm{CH}_{2} \mathrm{Cl}_{2}(0.8$ $\mathrm{mL}$ ) at $0{ }^{\circ} \mathrm{C}$ under argon and the mixture was stirred at room temperature for $1 \mathrm{~h}$. The mixture was diluted with EtOAc, washed with $1 \mathrm{~N} \mathrm{HCl,} \mathrm{water,} \mathrm{aqueous} \mathrm{saturated} \mathrm{NaHCO}_{3}$ and brine, dried over $\mathrm{Na}_{2} \mathrm{SO}_{4}$, and concentrated in vacuo. The residue was chromatographed on silica gel (hexane/EtOAc $=5 / 1)$ to afford $11 \mathrm{~b}\left(171 \mathrm{mg}, 81 \%, \mathrm{Z} / E=73 / 27\right.$, determined by $\left.{ }^{1} \mathrm{H} \mathrm{NMR}\right) .{ }^{11}$ Both products were isolated by column chromatography on silica gel followed by PTLC (silica gel) with hexane/EtOAc

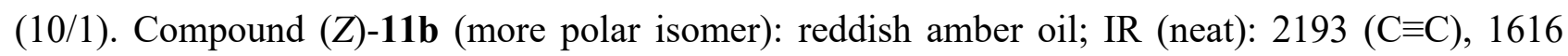
$(\mathrm{SiOC}=\mathrm{C}) ;{ }^{1} \mathrm{H} \mathrm{NMR}\left(500 \mathrm{MHz}, \mathrm{CDCl}_{3}\right) \delta: 0.26(\mathrm{~s}, 6 \mathrm{H}), 0.96(\mathrm{~s}, 9 \mathrm{H}), 1.54(\mathrm{t}, J=2.3 \mathrm{~Hz}, 3 \mathrm{H}), 2.41$ (s, 3H), 2.45 (dt, $J=7.4,7.4 \mathrm{~Hz}, 2 \mathrm{H}), 3.21(\mathrm{t}, J=7.4 \mathrm{~Hz}, 2 \mathrm{H}), 4.09$ (q, $J=2.3 \mathrm{~Hz}, 2 \mathrm{H}), 4.20$ (br s, 2H), $5.13(\mathrm{t}, J=7.4 \mathrm{~Hz}, 1 \mathrm{H}), 6.67-6.69(\mathrm{~m}, 2 \mathrm{H}), 7.12-7.13(\mathrm{~m}, 1 \mathrm{H}), 7.25-7.28(\mathrm{~m}, 3 \mathrm{H}), 7.73(\mathrm{~d}, J=$ 8.6 Hz, 2H); ${ }^{13} \mathrm{C}$ NMR (125 MHz, $\left.\mathrm{CDCl}_{3}\right) \delta:-4.1$ (2C), 3.2, 18.1, 21.5, 24.3, 25.7 (3C), 36.7, 45.3, 71.7, 81.5, 84.3, 92.1, 107.0, 114.3, 115.2, 117.8, 127.8 (2C), 129.2 (2C), 129.9, 131.9, 133.8, 
136.0, 143.1, 147.9; HRMS (FAB) calcd for $\mathrm{C}_{29} \mathrm{H}_{39} \mathrm{~N}_{2} \mathrm{O}_{3} \mathrm{SSi}\left(\mathrm{MH}^{+}\right)$523.2451, found 523.2458.

Compound (E)-11b (less polar isomer): reddish amber oil; IR (neat): $2193(\mathrm{C} \equiv \mathrm{C}), 1616(\mathrm{SiOC}=\mathrm{C})$; ${ }^{1} \mathrm{H}$ NMR $\left(500 \mathrm{MHz}, \mathrm{CDCl}_{3}\right) \delta: 0.23(\mathrm{~s}, 6 \mathrm{H}), 0.96(\mathrm{~s}, 9 \mathrm{H}), 1.51(\mathrm{t}, J=2.3 \mathrm{~Hz}, 3 \mathrm{H}), 2.40(\mathrm{~s}, 3 \mathrm{H}), 2.52$ $(\mathrm{dt}, J=7.6,7.6 \mathrm{~Hz}, 2 \mathrm{H}), 3.22(\mathrm{t}, J=7.6 \mathrm{~Hz}, 2 \mathrm{H}), 4.10(\mathrm{q}, J=2.3 \mathrm{~Hz}, 2 \mathrm{H}), 4.27(\mathrm{br} \mathrm{s}, 2 \mathrm{H}), 5.27(\mathrm{t}, J$ $=8.0 \mathrm{~Hz}, 1 \mathrm{H}), 6.66-6.70(\mathrm{~m}, 2 \mathrm{H}), 7.11-7.14(\mathrm{~m}, 1 \mathrm{H}), 7.25-7.29(\mathrm{~m}, 3 \mathrm{H}), 7.72(\mathrm{~d}, J=8.6 \mathrm{~Hz}, 2 \mathrm{H})$;

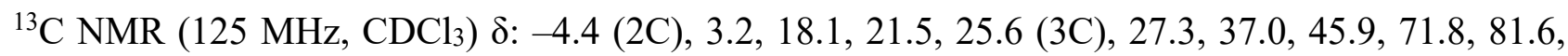
89.4, 89.7, 107.0, 114.4, 115.1, 117.7, 127.8 (2C), 129.2 (2C), 130.1, 132.1, 135.4, 136.0, 143.1, 148.0; HRMS (FAB) calcd for $\mathrm{C}_{29} \mathrm{H}_{39} \mathrm{~N}_{2} \mathrm{O}_{3} \mathrm{SSi}\left(\mathrm{MH}^{+}\right)$523.2451, found 523.2454.

\section{Gold(I)-Catalyzed Cascade Reactions.}

\section{General Procedure A: Synthesis of (R)-2,5-Diphenyl-4,5-dihydro-[1,4]oxazepino[4,5-} $a]$ indole (3a) and ( $R, Z)-1-B e n z y l i d e n e-4-p h e n y l-3,4-d i h y d r o-1 H-[1,4]$ oxazino[4,3-a]indole (4a). A screw-cap test tube was charged with $\mathbf{2 a}(33.7 \mathrm{mg}, 0.10 \mathrm{mmol}), \operatorname{IPrAuCl}(3.1 \mathrm{mg}, 5.0 \mu \mathrm{mol})$ and AgOTf $(1.3 \mathrm{mg}, 5.0 \mu \mathrm{mol})$. Dry 1,2-DCE $(1.5 \mathrm{~mL})$ was added to the screw-cap test tube. After stirring at $50{ }^{\circ} \mathrm{C}$ for $5 \mathrm{~h}$, the reaction mixture was concentrated in vacuo and chromatographed on $\mathrm{NH}_{2}$ silica gel (hexane/ $\mathrm{CHCl}_{3}=2 / 1$ ), and the collected solid was rinsed with hexane to afford an inseparable mixture of $\mathbf{3 a} / \mathbf{4 a}\left(29.0 \mathrm{mg}, 86 \%, \mathbf{3 a} / \mathbf{4 a}=71 / 29\right.$, determined by $\left.{ }^{1} \mathrm{H} \mathrm{NMR}\right)$ : greenish gray powder; mp $198-200{ }^{\circ} \mathrm{C}$; IR (neat) $1627\left(\mathrm{CH}_{2} \mathrm{OC}=\mathrm{C}\right) ;{ }^{1} \mathrm{H}$ NMR $\left(500 \mathrm{MHz}, \mathrm{CDCl}_{3}\right) \delta$ : (Major isomer 3a) $4.70(\mathrm{dd}, J=12.6,1.1 \mathrm{~Hz}, 1 \mathrm{H}), 5.00(\mathrm{dd}, J=12.6,3.7 \mathrm{~Hz}, 1 \mathrm{H}), 5.89$ (br m, 1H), 6.47 (s, 1H), $6.55(\mathrm{~s}, 1 \mathrm{H}), 7.04-7.08(\mathrm{~m}, 3 \mathrm{H}), 7.11-7.17(\mathrm{~m}, 2 \mathrm{H}), 7.19-7.35(\mathrm{~m}, 6 \mathrm{H}), 7.58-7.58(\mathrm{~m}, 1 \mathrm{H})$, 7.63-7.64 (m, 2H); (Minor isomer 4a): 4.57-4.61 (m, 2H), 5.50-5.50 (br m, 1H), 6.37 (s, 1H), 6.94 (s, 1H), $6.98(\mathrm{~d}, J=8.0 \mathrm{~Hz}, 1 \mathrm{H}), 7.11-7.17(\mathrm{~m}, 5 \mathrm{H}), 7.19-7.35(\mathrm{~m}, 6 \mathrm{H}), 7.69(\mathrm{~d}, J=7.4 \mathrm{~Hz}, 2 \mathrm{H})$; ${ }^{13} \mathrm{C}$ NMR (125 MHz, $\mathrm{CDCl}_{3}$ ) $\delta$ : (Major isomer 3a): 61.3, 73.3, 96.6, 102.8, 109.1, 120.19, 120.20, $121.7,125.5$ (2C), 126.5 (2C), 127.7, 128.2 (2C), 128.3, 128.5, 128.6 (2C), 136.0, 136.6, 138.1, 138.4, 153.4; (Minor isomer 4a): 55.3, 70.6, 97.7, 105.5, 109.6, 120.8 (2C), 122.2 (2C), 126.1 (2C), 
$127.8,128.2$ (2C), 128.3, 128.46, 128.47, 128.7, 128.9, 130.6, 135.7, 135.9, 138.1, 144.7; HRMS (ESI) calcd for $\mathrm{C}_{24} \mathrm{H}_{20} \mathrm{NO}\left(\mathrm{MH}^{+}\right)$: 338.1545; found: 338.1550 .

$( \pm)-(4 R, 5 S)-2,4,5-T r i p h e n y l-4,5-d i h y d r o-[1,4]$ oxazepino[4,5-a]indole $(3 \mathrm{~b})$ and $( \pm)-(3 R, 4 S)-$ 1-[(Z)-Benzylidene]-3,4-diphenyl-3,4-dihydro-1H-[1,4]oxazino[4,3-a]indole (4b). By use of the General Procedure A, 2b (41.4 mg, $0.10 \mathrm{mmol})$ was converted to $\mathbf{3 b} / \mathbf{4 b}(37.9 \mathrm{mg}, 92 \%, \mathbf{3 b} / \mathbf{4 b}=$ 29/71, determined by ${ }^{1} \mathrm{H}$ NMR) by the reaction in the presence of $\mathrm{IPrAuCl}(3.1 \mathrm{mg}, 5.0 \mu \mathrm{mol})$ and $\operatorname{AgOTf}(1.3 \mathrm{mg}, 5.0 \mu \mathrm{mol})$ in dry $1,2-\mathrm{DCE}(1.0 \mathrm{~mL})$ at $50{ }^{\circ} \mathrm{C}$ for $2.5 \mathrm{~h}$. Both the products were isolated by PTLC ( $\mathrm{NH}_{2}$ silica gel) with hexane/Et $2 \mathrm{O}$ (3/1). Compound $\mathbf{3 b}$ (less polar isomer): white solid; $\mathrm{mp}>250{ }^{\circ} \mathrm{C}$; IR (neat): $1642(\mathrm{CHOC}=\mathrm{C}) ;{ }^{1} \mathrm{H}$ NMR $\left(500 \mathrm{MHz}, \mathrm{CDCl}_{3}\right) \delta: 5.90(\mathrm{~d}, J=6.9 \mathrm{~Hz}$, 2H), $6.61(\mathrm{~s}, 1 \mathrm{H}), 6.64(\mathrm{~s}, 1 \mathrm{H}), 6.82(\mathrm{~d}, J=6.9 \mathrm{~Hz}, 2 \mathrm{H}), 7.05-7.12(\mathrm{~m}, 5 \mathrm{H}), 7.15-7.18(\mathrm{~m}, 1 \mathrm{H})$, 7.23-7.24 (m, 2H), 7.32-7.36 (m, 6H), 7.59-7.60 (m, 1H), 7.69-7.70 (m, 2H); ${ }^{13} \mathrm{C}$ NMR (125 MHz, $\left.\mathrm{CDCl}_{3}\right) \delta: 67.5,83.4,97.2,103.0,109.2,120.19,120.24,121.8,125.7$ (2C), $126.6(2 \mathrm{C}), 127.71$ (2C), 127.73, 127.9, 128.0, 128.25 (2C), $128.34(2 \mathrm{C}), 128.4(2 \mathrm{C}), 128.6,135.7,135.9,136.4,138.1$, 138.4, 152.7; HRMS (FAB) calcd for $\mathrm{C}_{30} \mathrm{H}_{24} \mathrm{NO}\left(\mathrm{MH}^{+}\right)$414.1852, found 414.1861. Compound 4b (more polar isomer): pale yellow solid; mp $168-172{ }^{\circ} \mathrm{C}$; IR (neat): $1632\left(\mathrm{CH}_{2} \mathrm{OC}=\mathrm{C}\right) ;{ }^{1} \mathrm{H}$ NMR

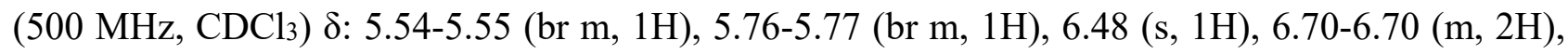
$7.00(\mathrm{~d}, J=8.6 \mathrm{~Hz}, 1 \mathrm{H}), 7.04-7.05(\mathrm{~m}, 2 \mathrm{H}), 7.09-7.18(\mathrm{~m}, 7 \mathrm{H}), 7.26-7.33(\mathrm{~m}, 5 \mathrm{H}), 7.65-7.67(\mathrm{~m}$, 1H), $7.75(\mathrm{~d}, J=7.4 \mathrm{~Hz}, 2 \mathrm{H}) ;{ }^{13} \mathrm{C} \mathrm{NMR}\left(125 \mathrm{MHz}, \mathrm{CDCl}_{3}\right) \delta: 60.9,80.3,97.5,105.8,109.1,120.82$, $120.84,122.3,126.1,126.5$ (2C), 127.881 (2C), $127.886(2 \mathrm{C}), 128.0,128.17(2 \mathrm{C}), 128.22,128.35$ (2C), 128.38, 128.8 (2C), 130.3, 134.8, 135.6, 135.7, 136.2, 144.9; HRMS (FAB) calcd for $\mathrm{C}_{30} \mathrm{H}_{24} \mathrm{NO}\left(\mathrm{MH}^{+}\right)$414.1852, found 414.1859.

\section{5-Phenyl-2-propyl-[1,4]oxazepino[4,5-a]indol-4(5H)-one $(3 \mathrm{c})$ and (Z)-1-Butylidene-4-} phenyl-1H-[1,4]oxazino[4,3-a]indol-3(4H)-one (4c). By use of the General Procedure A, 2c (31.7 $\mathrm{mg}, 0.10 \mathrm{mmol})$ was converted to $\mathbf{3 c} / \mathbf{4 c}\left(11.1 \mathrm{mg},<35 \%, \mathbf{3 c} / \mathbf{4} \mathbf{c}=20 / 80\right.$, determined by $\left.{ }^{1} \mathrm{H} \mathrm{NMR}\right)$ by the reaction in the presence of $\operatorname{IPrAuCl}(3.1 \mathrm{mg}, 5.0 \mu \mathrm{mol})$ and $\mathrm{AgOTf}(1.3 \mathrm{mg}, 5.0 \mu \mathrm{mol})$ in dry 1,2-DCE $(1.0 \mathrm{~mL})$ at $50{ }^{\circ} \mathrm{C}$ for $3 \mathrm{~h}$. The products were separated by PTLC $\left(\mathrm{NH}_{2}\right.$ silica gel) with 
hexane/ $\mathrm{Et}_{2} \mathrm{O}$ (3/1). Compound 3c (less polar isomer): unstable pale amber oil; IR (neat): 1749 $(\mathrm{C}=\mathrm{O}) ;{ }^{1} \mathrm{H}$ NMR $\left(500 \mathrm{MHz}, \mathrm{CDCl}_{3}\right) \delta: 0.62(\mathrm{t}, J=7.4 \mathrm{~Hz}, 3 \mathrm{H}), 1.10-1.19(\mathrm{~m}, 1 \mathrm{H}), 1.33-1.43(\mathrm{~m}$, 1H), 1.95-2.01 (m, 1H), 2.11-2.17 (m, 1H), $5.96(\mathrm{~s}, 1 \mathrm{H}), 6.55(\mathrm{~s}, 1 \mathrm{H}), 6.67-6.69(\mathrm{~m}, 2 \mathrm{H}), 6.76(\mathrm{~s}$, 1H), 7.19-7.29 (m, 5H), $7.43(\mathrm{~d}, J=8.0 \mathrm{~Hz}, 1 \mathrm{H}), 7.67(\mathrm{~d}, J=7.4 \mathrm{~Hz}, 1 \mathrm{H}) ;{ }^{13} \mathrm{C} \mathrm{NMR}(125 \mathrm{MHz}$, $\left.\mathrm{CDCl}_{3}\right) \delta: 13.0,19.9,37.0,62.9,102.3,102.9,108.7,120.8,121.1,122.6,124.6(2 \mathrm{C}), 128.5,128.9$ (3C), 132.3, 133.0, 136.9, 149.0, 164.2; HRMS (FAB) calcd for $\mathrm{C}_{21} \mathrm{H}_{20} \mathrm{NO}_{2}\left(\mathrm{MH}^{+}\right)$318.1489, found 318.1484. Compound $4 \mathbf{c}$ (more polar isomer): unstable yellow oil; IR (neat): $1760(\mathrm{C}=\mathrm{O})$; ${ }^{1} \mathrm{H}$ NMR $\left(500 \mathrm{MHz}, \mathrm{CDCl}_{3}\right) \delta: 0.93(\mathrm{t}, J=7.4 \mathrm{~Hz}, 3 \mathrm{H}), 1.47-1.51(\mathrm{~m}, 2 \mathrm{H}), 2.29-2.41(\mathrm{~m}, 2 \mathrm{H}), 5.69(\mathrm{t}, J=7.7$ $\mathrm{Hz}, 1 \mathrm{H}), 6.24(\mathrm{~s}, 1 \mathrm{H}), 6.82(\mathrm{~s}, 1 \mathrm{H}), 7.04-7.06(\mathrm{~m}, 3 \mathrm{H}), 7.10-7.17(\mathrm{~m}, 2 \mathrm{H}), 7.28-7.28(\mathrm{~m}, 3 \mathrm{H}), 7.63$ $(\mathrm{d}, J=7.4 \mathrm{~Hz}, 1 \mathrm{H}) ;{ }^{13} \mathrm{C} \mathrm{NMR}\left(125 \mathrm{MHz}, \mathrm{CDCl}_{3}\right) \delta: 13.7,22.4,26.6,59.2,97.5,109.7,112.4,121.0$, $121.4,122.8,126.1$ (2C), 127.1, 129.0, 129.1, 129.2 (2C), 134.65, 134.74, 139.5, 163.1; HRMS (FAB) calcd for $\mathrm{C}_{21} \mathrm{H}_{20} \mathrm{NO}_{2}\left(\mathrm{MH}^{+}\right)$318.1489, found 318.1484.

\section{(Z)-N-(But-2-yn-1-yl)-4-methyl-N-[3-(3-oxo-4-phenyl-3,4-dihydro-1H-[1,4]oxazino[4,3-}

a]indol-1-ylidene)propyl]benzenesulfonamide (20b). A screw-cap test tube was charged with ( \pm )$10 b$ (52.5 mg, $0.1 \mathrm{mmol}), \mathrm{IPrAuCl}(6.2 \mathrm{mg}, 0.01 \mathrm{mmol})$ and $\operatorname{AgOTf}(2.6 \mathrm{mg}, 0.01 \mathrm{mmol})$. Dry 1,2DCE $(1 \mathrm{~mL})$ was added to the screw-cap test tube. After stirring at $50{ }^{\circ} \mathrm{C}$ for $27 \mathrm{~h}$, the mixture was concentrated in vacuo and chromatographed on $\mathrm{NH}_{2}$ silica gel (hexane $/ \mathrm{CHCl}_{3}=3 / 1$ ) to afford 20b/20b' (16.3 mg, <31\%, 20b/20b' = 90/10, determined by ${ }^{1} \mathrm{H}$ NMR) as an isomeric mixture of unstable compounds. The major isomer $20 \mathrm{~b}$ was isolated by PTLC (silica gel) with hexane/Et ${ }_{2} \mathrm{O}$ (3/1): unstable yellow oil; IR (neat): $2225(\mathrm{C} \equiv \mathrm{C}), 1765(\mathrm{C}=\mathrm{O}), 1345(\mathrm{~S}=\mathrm{O}), 1157(\mathrm{~S}=\mathrm{O}) ;{ }^{1} \mathrm{H}$ NMR $\left(500 \mathrm{MHz} \mathrm{CDCl}_{3}\right) \delta: 1.53(\mathrm{t}, J=2.3 \mathrm{~Hz}, 3 \mathrm{H}), 2.40(\mathrm{~s}, 3 \mathrm{H}), 2.61-2.65(\mathrm{~m}, 2 \mathrm{H}), 3.33-3.34(\mathrm{~m}, 2 \mathrm{H})$, 3.99-4.10 (m, 2H), $5.73(\mathrm{t}, J=7.4 \mathrm{~Hz}, 1 \mathrm{H}), 6.25(\mathrm{~s}, 1 \mathrm{H}), 6.87(\mathrm{~s}, 1 \mathrm{H}), 7.05-7.07(\mathrm{~m}, 3 \mathrm{H}), 7.11-7.17$ (m, 2H), 7.27-7.30 (m, 5H), 7.64-7.66 (m, 1H), $7.72(\mathrm{~m}, 2 \mathrm{H}) ;{ }^{13} \mathrm{C} \mathrm{NMR}\left(125 \mathrm{MHz}, \mathrm{CDCl}_{3}\right) \delta: 3.2$, 21.5, 23.0, 36.7, 45.2, 59.2, 71.5, 81.9, 98.5, 107.6, 109.7, 121.3, 121.5, 123.06, 126.13 (2C), 126.4, 127.8 (2C), 128.9, 129.2, 129.27 (2C), 129.31 (2C), 134.65, 134.71, 135.9, 140.9, 143.3, 162.8; HRMS (FAB) calcd for $\mathrm{C}_{31} \mathrm{H}_{29} \mathrm{~N}_{2} \mathrm{O}_{4} \mathrm{~S}\left(\mathrm{MH}^{+}\right)$525.1848, found 525.1851 . 


\section{Gold(I)-Catalyzed Cyclization of the Conjugated Enyne (Table 1): (E)-(3-Ethylidene-1-} tosylpiperidin-4-yl)(1H-indol-2-yl)methanone $[( \pm)-9]$. The experiments shown in Table 1 were carried out as follows: 11a $(56.5 \mathrm{mg}, 0.1 \mathrm{mmol} ; Z / E=79: 21)$ or $11 \mathbf{b}(52.3 \mathrm{mg}, 0.1 \mathrm{mmol} ; Z / E=$ 71:29) were treated with JohnPhosAu(MeCN)SbF6 (3.9 mg, 5.0 $\mu \mathrm{mol})$ or IPrAuCl (3.1 mg, 5.0/5.0 $\mu \mathrm{mol} ; 5 \mathrm{~mol} \%) / \operatorname{AgSbF}_{6}(1.7 \mathrm{mg}, 5.0 \mu \mathrm{mol} ; 5 \mathrm{~mol} \%)$ in toluene- $d_{8}(0.5 \mathrm{~mL}, 0.2 \mathrm{M})$ at room temperature in the presence of an additive (1.5 equiv) and mesitylene (1.0 equiv) as an internal standard. After completion of the reaction (monitored by TLC), the reaction mixtures were analyzed by ${ }^{1} \mathrm{H}$ NMR to determine the yields of $( \pm)-\mathbf{9}, \mathbf{2 8}$, and 29 based on the internal standard. Pure $( \pm)-9$ was obtained as follows: a screw-cap test tube was charged with 11a $(293 \mathrm{mg}, 0.52 \mathrm{mmol}, \mathrm{Z} / \mathrm{E}=$ 93/7) and JohnPhosAu(MeCN)SbF 6 (20 mg, $25.9 \mu \mathrm{mol}) . \mathrm{H}_{2} \mathrm{O}(14 \mu \mathrm{L}, 0.8 \mathrm{mmol})$ and dry toluene $(2.6 \mathrm{~mL})$ were added to the mixture. After stirring at room temperature for $24 \mathrm{~h}$, the mixture was concentrated in vacuo and chromatographed on $\mathrm{NH}_{2}$ silica gel (hexane/EtOAc $=5 / 1$ ). The product was recrystallized from $\mathrm{CHCl}_{3}-$ hexane to afford $( \pm)-9(64.1 \mathrm{mg}, 30 \%)$ as white solid: $\mathrm{mp} 183-$ $186{ }^{\circ} \mathrm{C}$; IR (neat): $3343(\mathrm{NH}), 1643(\mathrm{C}=\mathrm{O}), 1341(\mathrm{~S}=\mathrm{O}), 1162(\mathrm{~S}=\mathrm{O}) ;{ }^{1} \mathrm{H}$ NMR $\left(500 \mathrm{MHz}, \mathrm{CDCl}_{3}\right)$ $\delta: 1.72(\mathrm{dd}, J=6.9,1.7 \mathrm{~Hz}, 3 \mathrm{H}), 2.06-2.11(\mathrm{~m}, 1 \mathrm{H}), 2.15-2.16(\mathrm{~m}, 1 \mathrm{H}), 2.44(\mathrm{~s}, 3 \mathrm{H}), 2.86(\mathrm{ddd}, J=$ 12.2, 12.0, 3.2 Hz, 1H), 3.43-3.45 (br m, 1H), 3.71-3.74 (br m, 1H), 4.09-4.11 (br m, 1H), 4.43-4.43 (br m, 1H), $5.72(\mathrm{q}, J=6.7 \mathrm{~Hz}, 1 \mathrm{H}), 7.14-7.17(\mathrm{~m}, 1 \mathrm{H}), 7.25(\mathrm{~d}, J=2.3 \mathrm{~Hz}, 1 \mathrm{H}), 7.33-7.36(\mathrm{~m}, 4 \mathrm{H})$, $7.66(\mathrm{~d}, J=8.0 \mathrm{~Hz}, 2 \mathrm{H}), 7.70(\mathrm{~d}, J=9.2 \mathrm{~Hz}, 1 \mathrm{H}), 8.86($ br s, $1 \mathrm{H}) ;{ }^{13} \mathrm{C} \mathrm{NMR}\left(125 \mathrm{MHz}, \mathrm{CDCl}_{3}\right) \delta$ : $13.3,21.6,28.5,41.2,43.2,52.3,109.4,112.0,121.2,123.2,124.8,126.7,127.4,127.7$ (2C), 129.7 (2C), 129.9, 133.5, 133.8, 137.3, 143.5, 192.6; HRMS (FAB) calcd for $\mathrm{C}_{23} \mathrm{H}_{25} \mathrm{~N}_{2} \mathrm{O}_{3} \mathrm{~S}\left(\mathrm{MH}^{+}\right)$ 409.1586, found 409.1577.

\section{(Z)-N-\{4-(1H-Indol-2-yl)-4-[(triisopropylsilyl)oxy]but-3-en-1-yl\}-N-(but-2-yn-1-yl)-4-}

methylbenzenesulfonamide (28). Amber oil: IR (neat): $3386(\mathrm{NH}), 2225(\mathrm{C} \equiv \mathrm{C}), 1650(\mathrm{SiOC}=\mathrm{C})$, $1341(\mathrm{~S}=\mathrm{O}), 1157(\mathrm{~S}=\mathrm{O}) ;{ }^{1} \mathrm{H}$ NMR $\left(500 \mathrm{MHz}, \mathrm{CDCl}_{3}\right) \delta: 1.09(\mathrm{~d}, J=6.9 \mathrm{~Hz}, 18 \mathrm{H}), 1.18-1.21(\mathrm{~m}$, $3 \mathrm{H}), 1.56(\mathrm{t}, J=2.3 \mathrm{~Hz}, 3 \mathrm{H}), 2.40(\mathrm{~s}, 3 \mathrm{H}), 2.54(\mathrm{q}, J=7.3 \mathrm{~Hz}, 2 \mathrm{H}), 3.27(\mathrm{t}, J=7.4 \mathrm{~Hz}, 2 \mathrm{H}), 4.11(\mathrm{q}$, $J=2.3 \mathrm{~Hz}, 2 \mathrm{H}), 5.13(\mathrm{t}, J=6.9 \mathrm{~Hz}, 1 \mathrm{H}), 6.54-6.54$ (br m, 1H), 7.08-7.10 (m, 1H), 7.16-7.18 (m, 
1H), $7.26(\mathrm{~d}, J=8.0 \mathrm{~Hz}, 2 \mathrm{H}), 7.33(\mathrm{~d}, J=8.0 \mathrm{~Hz}, 1 \mathrm{H}), 7.57(\mathrm{~d}, J=7.4 \mathrm{~Hz}, 1 \mathrm{H}), 7.74(\mathrm{~d}, J=8.6 \mathrm{~Hz}$, 2H), 8.15 (br s, $1 \mathrm{H}) ;{ }^{13} \mathrm{C}$ NMR (125 MHz, $\left.\mathrm{CDCl}_{3}\right) \delta: 3.3,13.6$ (3C), 17.9 (6C), 21.5, 24.5, 36.8, $45.8,71.8,81.5,100.1,106.4,110.8,119.9,120.6,122.2,127.8(2 \mathrm{C}), 128.5,129.2(2 \mathrm{C}), 135.7$, 136.0, 136.7, 143.2, 144.8; HRMS (FAB) calcd for $\mathrm{C}_{32} \mathrm{H}_{45} \mathrm{~N}_{2} \mathrm{O}_{3} \mathrm{SSi}\left(\mathrm{MH}^{+}\right)$565.2920, found 565.2927.

\section{$N$-[4-(1H-Indol-2-yl)-4-oxobutyl]-N-(but-2-yn-1-yl)-4-methylbenzenesulfonamide}

White powder: $\mathrm{mp} 120-121^{\circ} \mathrm{C}$; IR (neat): $3326(\mathrm{NH}), 2224(\mathrm{C} \equiv \mathrm{C}), 1649(\mathrm{C}=\mathrm{O}), 1340(\mathrm{~S}=\mathrm{O}), 1157$ $(\mathrm{S}=\mathrm{O}) ;{ }^{1} \mathrm{H}$ NMR $\left(500 \mathrm{MHz}, \mathrm{CDCl}_{3}\right) \delta: 1.53(\mathrm{t}, J=2.3 \mathrm{~Hz}, 3 \mathrm{H}), 2.01-2.07(\mathrm{~m}, 2 \mathrm{H}), 2.41(\mathrm{~s}, 3 \mathrm{H})$, $3.08(\mathrm{t}, J=7.4 \mathrm{~Hz}, 2 \mathrm{H}), 3.30(\mathrm{t}, J=6.9 \mathrm{~Hz}, 2 \mathrm{H}), 4.08-4.08$ (br m, 2H), 7.15-7.17 (m, 1H), 7.25-7.28 (m, 3H), 7.34-7.36 (m, 1H), 7.41-7.43 (m, 1H), 7.72-7.74 (m, 3H), 9.01 (br s, $1 \mathrm{H}) ;{ }^{13} \mathrm{C}$ NMR $(125$ $\left.\mathrm{MHz}, \mathrm{CDCl}_{3}\right) \delta: 3.2,21.4,22.0,34.9,36.9,45.8,71.7,81.7,109.4,112.1,120.9,123.1,126.3$, 127.6, 127.8 (2C), 129.3 (2C), 134.9, 135.9, 137.1, 143.3, 192.2; HRMS (FAB) calcd for $\mathrm{C}_{23} \mathrm{H}_{25} \mathrm{~N}_{2} \mathrm{O}_{3} \mathrm{~S}\left(\mathrm{MH}^{+}\right)$409.1586, found 409.1590.

Enantioselective Cyclization of Conjugated Enyne (Table 2): Synthesis of (S,E)-(3-

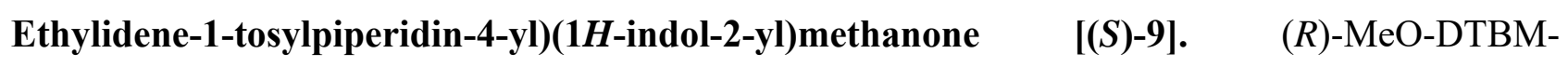
$\operatorname{BIPHEP}(\mathrm{AuCl})_{2}(8.1 \mathrm{mg}, 5.0 \mu \mathrm{mol} ; 5 \mathrm{~mol} \%)$ and $\mathrm{AgSbF}_{6}(3.4 \mathrm{mg}, 0.01 \mathrm{mmol} ; 10 \mathrm{~mol} \%)$ was dissolved in toluene $(0.1 \mathrm{~mL})$ and stirred for $10 \mathrm{~min}$ at room temperature. A solution of $(Z)-11 a$ ( $56.5 \mathrm{mg}, 0.1 \mathrm{mmol})$ in toluene $(0.4 \mathrm{~mL})$ was transferred to the catalyst mixture. The mixture was stirred at room temperature for $17 \mathrm{~h}$. The mixture was concentrated and purified on PTLC (silica gel) with hexane/EtOAc (3/1) to afford (S)-9 as a white amorphous solid $\{13.1 \mathrm{mg}, 32 \%$ yield, $88 \%$ ee [HPLC, Chiralcel-OD-H column eluting under condition with $40 \%$ i-PrOH/n-hexane over 30 $\min$ at $0.75 \mathrm{~mL} / \mathrm{min}, t_{1}=12.66 \mathrm{~min}$ (major isomer), $t_{2}=16.60 \mathrm{~min}$ (minor isomer) $\left.]\right\}:[\alpha]^{26} \mathrm{D}-2.1(c$ 0.92, $\left.\mathrm{CHCl}_{3}\right)$; IR (neat): $3334(\mathrm{NH}), 1641(\mathrm{C}=\mathrm{O}), 1341(\mathrm{~S}=\mathrm{O}), 1159(\mathrm{~S}=\mathrm{O}) ;{ }^{1} \mathrm{H}$ NMR $(500 \mathrm{MHz}$, $\left.\mathrm{CDCl}_{3}\right) \delta: 1.71(\mathrm{dd}, J=6.9,1.7 \mathrm{~Hz}, 3 \mathrm{H}), 2.03-2.10(\mathrm{~m}, 1 \mathrm{H}), 2.14-2.17(\mathrm{~m}, 1 \mathrm{H}), 2.40(\mathrm{~s}, 3 \mathrm{H}), 2.88$ (ddd, $J=12.3,12.3,2.9 \mathrm{~Hz}, 1 \mathrm{H}), 3.47-3.49$ (br m, 1H), 3.71-3.74 (br m, 1H), 4.10-4.13 (br m, 1H), 4.43-4.44 (br m, 1H), 5.71 (q, $J=6.9 \mathrm{~Hz}, 1 \mathrm{H}), 7.13-7.16(\mathrm{~m}, 1 \mathrm{H}), 7.32-7.34(\mathrm{~m}, 4 \mathrm{H}), 7.65(\mathrm{~d}, J=$ 
$8.0 \mathrm{~Hz}, 2 \mathrm{H}), 7.70(\mathrm{~d}, J=8.0 \mathrm{~Hz}, 1 \mathrm{H}), 9.20($ br s, $1 \mathrm{H}) ;{ }^{13} \mathrm{C} \mathrm{NMR}\left(125 \mathrm{MHz}, \mathrm{CDCl}_{3}\right) \delta: 13.3,21.5$, $28.5,41.1,43.2,52.3,109.5,112.1,121.2,123.1,124.7,126.7,127.4,127.7$ (2C), 129.6 (2C), 129.9, 133.4, 133.8, 137.3, 143.4, 192.7; HRMS (FAB) calcd for $\mathrm{C}_{23} \mathrm{H}_{25} \mathrm{~N}_{2} \mathrm{O}_{3} \mathrm{~S}\left(\mathrm{MH}^{+}\right)$409.1586, found 409.1585 .

\section{Total Synthesis of (+)-Conolidine (Scheme 6).}

(S,E)-(3-Ethylidenepiperidin-4-yl)(1H-indol-2-yl)methanone (7). According to the reported method, ${ }^{26}$ removal of the tosyl group was carried out as follows: sodium $(27.7 \mathrm{mg}, 1.20 \mathrm{mmol})$ was added to a solution of naphthalene $(193 \mathrm{mg}, 1.50 \mathrm{mmol})$ in THF $(1.5 \mathrm{~mL})$ at room temperature and the mixture stirred for $30 \mathrm{~min}$. The resulting dark green/blue solution (ca. $0.8 \mathrm{M}$ in THF) was added dropwise to a solution of (S)-9 (49.2 mg, $0.12 \mathrm{mmol}, 91 \%$ ee) in THF $(1.2 \mathrm{~mL})$ at $0{ }^{\circ} \mathrm{C}$ until dark green/blue color persisted. Saturated aqueous $\mathrm{NaHCO}_{3}$ was added and the solution was allowed to warm slowly to room temperature. The aqueous layer was then extracted with $\mathrm{CH}_{2} \mathrm{Cl}_{2}$ and the organic layers were washed with brine, combined, dried over $\mathrm{K}_{2} \mathrm{CO}_{3}$ and filtered. Concentration under reduced pressure and recrystallization from $\mathrm{CH}_{2} \mathrm{Cl}_{2}$-hexane afforded (+)-7 (18.5 mg, 60\%): mp 205-210 ${ }^{\circ} \mathrm{C} ;[\alpha]^{28}{ }_{\mathrm{D}}=+41.0\left(\right.$ c 0.26, MeOH), comparable to the report by Bohn et. al. $(2011)^{16}$ : $[\alpha]^{25} \mathrm{D}=+45.0\left(\right.$ c 0.24, MeOH); IR (neat): $3345(\mathrm{NH}), 1627(\mathrm{C}=\mathrm{O}) ;{ }^{1} \mathrm{H}$ NMR $\left(500 \mathrm{MHz}, \mathrm{CDCl}_{3}\right) \delta$ : $1.72(\mathrm{dd}, J=6.9,1.7 \mathrm{~Hz}, 3 \mathrm{H}), 1.87-1.95(\mathrm{~m}, 1 \mathrm{H}), 2.20-2.22$ (br m, 1H), 2.97-3.00 (br m, $1 \mathrm{H}), 3.15$ (ddd, $J=12.6,12.6,2.9 \mathrm{~Hz}, 1 \mathrm{H}), 3.33$ (d, $J=12.6 \mathrm{~Hz}, 1 \mathrm{H}), 3.49$ (s, 1H), 3.72 (d, $J=12.6 \mathrm{~Hz}, 1 \mathrm{H})$, 4.53-4.54 (br m, 1H), 5.54 (q, $J=6.7 \mathrm{~Hz}, 1 \mathrm{H}), 7.15-7.17(\mathrm{~m}, 1 \mathrm{H}), 7.28(\mathrm{~s}, 1 \mathrm{H}), 7.34-7.36(\mathrm{~m}, 1 \mathrm{H})$, $7.43(\mathrm{~d}, J=8.0 \mathrm{~Hz}, 1 \mathrm{H}), 7.71(\mathrm{~d}, J=8.0 \mathrm{~Hz}, 1 \mathrm{H}), 9.16($ br s, $1 \mathrm{H}) ;{ }^{13} \mathrm{C} \mathrm{NMR}\left(125 \mathrm{MHz}, \mathrm{CDCl}_{3}\right) \delta$ : $13.0,31.5,43.0,43.2,53.1,108.9,112.1,120.8,121.0,123.1,126.3,127.6,134.4,135.1,137.1$, 193.6; HRMS (FAB) calcd for $\mathrm{C}_{16} \mathrm{H}_{19} \mathrm{~N}_{2} \mathrm{O}\left(\mathrm{MH}^{+}\right)$255.1497, found 255.1490.

(+)-Conolidine (1). According to the Bohn's procedure, ${ }^{2}$ the amine $(+)-7(16.0 \mathrm{mg}, 0.06 \mathrm{mmol})$, paraformaldehyde $(6.8 \mathrm{mg}, 0.23 \mathrm{mmol})$, and TFA $(14.5 \mu \mathrm{L}, 0.19 \mathrm{mmol})$ were dissolved in dry $\operatorname{MeCN}(1.2 \mathrm{~mL})$ and the reaction mixture heated under reflux for $2 \mathrm{~h}$. TFA (14.5 $\mu \mathrm{L})$ was added and 
the mixture was stirred for further $3 \mathrm{~h}$. The mixture was concentrated in vacuo and the crude product was made basic with aqueous saturated $\mathrm{NaHCO}_{3}$ (to $\mathrm{pH}$ 9.0) and extracted with $\mathrm{CH}_{2} \mathrm{Cl}_{2}$ three times. The resultant orange solution was dried over $\mathrm{Na}_{2} \mathrm{SO}_{4}$, concentrated in vacuo and chromatographed on $\mathrm{NH}_{2}$ silica gel $\left(\mathrm{MeOH} / \mathrm{CHCl}_{3}=99 / 1\right)$ to afford $(+)$-conolidine (1) (5.7 mg, $34 \%, 84 \%$ ee [HPLC, Chiralcel-AD-H column eluting under condition with $80 \% i$-PrOH/n-hexane over $25 \mathrm{~min}$ at $0.75 \mathrm{~mL} / \mathrm{min}, t_{1}=14.25 \mathrm{~min}$ (minor isomer), $t_{2}=16.95 \mathrm{~min}$ (major isomer)]): $\mathrm{mp}$ $178-180{ }^{\circ} \mathrm{C} ;[\alpha]^{28} \mathrm{D}=+31.5\left(c 0.24, \mathrm{CHCl}_{3}\right)$, comparable to the report by Kam et. al. $(2004)^{1}:[\alpha]_{\mathrm{D}}=$ +32.0 (c 0.16, $\left.\mathrm{CHCl}_{3}\right)$, Bohn et. al. $(2011)^{16}:[\alpha]^{27} \mathrm{D}=+28.1$ (c 0.16, $\left.\mathrm{CHCl}_{3}\right)$; IR (neat): 2914 (NH), $1634(\mathrm{C}=\mathrm{O}) ;{ }^{1} \mathrm{H}$ NMR $\left(500 \mathrm{MHz}, \mathrm{CDCl}_{3}\right) \delta$ : 1.51-1.52 (br m, 3H), 2.04-2.06 (m, 1H), 2.10-2.18 (m, 1H), 3.06-3.13 (m, 1H), 3.30-3.33 (br m, 1H), 3.41 (ddd, $J=13.7,8.6,2.9 \mathrm{~Hz}, 1 \mathrm{H}), 3.85-3.88$ (br m, 1H), 3.97-3.98 (br m, 1H), 4.29 (d, $J=18.3 \mathrm{~Hz}, 1 \mathrm{H}), 4.78$ (d, $J=18.3 \mathrm{~Hz}, 1 \mathrm{H}), 5.47$ (q, $J=6.9 \mathrm{~Hz}$, 1H), 7.11 (ddd, $J=16.0,8.0,4.0 \mathrm{~Hz}, 1 \mathrm{H}), 7.32-7.37(\mathrm{~m}, 2 \mathrm{H}), 7.57$ (d, $J=8.0 \mathrm{~Hz}, 1 \mathrm{H}), 9.02$ (br s, $1 \mathrm{H}) ;{ }^{13} \mathrm{C} \mathrm{NMR}\left(125 \mathrm{MHz}, \mathrm{CDCl}_{3}\right) \delta: 12.7,22.9,44.2,48.1,53.3,55.0,111.7,120.1,120.5,120.8$, 122.9, 126.5, 127.9, 130.1, 133.5, 136.1, 193.5; HRMS (FAB) calcd for $\mathrm{C}_{17} \mathrm{H}_{19} \mathrm{~N}_{2} \mathrm{O}\left(\mathrm{MH}^{+}\right)$ 267.1497, found 267.1494 .

Acknowledgments This work was supported by a Grant-in-Aid for Encouragement of Young Scientists (A) (H.O.), Scientific Research on Innovative Areas "Integrated Organic Synthesis" (H.O.) and the Targeted Proteins Research Program from the Ministry of Education, Culture, Sports, Science and Technology of Japan. Y.S. is grateful for Research Fellowships from the JSPS for Young Scientists.

\section{Supporting Information Available.}

Additional synthetic schemes (preparation of $\mathbf{2 a - c}$ ), NMR spectra, and HPLC chromatograms. This material is available freely via the Internet at http://pubs.acs.org. 


\section{References}

(1) Kam, T.-S.; Pand, H.-S.; Choo, Y.-M.; Komiyama, K. Chem. Biodivers. 2004, 1, 646.

(2) Tarselli, M. A.; Raehal, K. M.; Brasher, A. K.; Streicher, J. M.; Groer, C. E.; Cameron, M. D.; Bohn, L. M.; Micalizio, G. C. Nat. Chem. 2011, 3, 449.

(3) (a) Bennasar, M.-L.; Zulaica, E.; Solé, D.; Alonso, S. Chem. Commun. 2009, 3372. (b) Bennasar, M.-L.; Zulaica, E.; Solé, D.; Roca, T.; García-Díaz, D.; Alonso, S. J. Org. Chem. 2009, 74, 8359. (c) Hirose, T.; Noguchi, Y.; Furuya, Y.; Ishiyama, A.; Iwatsuki, M.; Otoguro, K.; Ōmura, S.; Sunazuka, T. Chem. Eur. J. 2013, 19, 10741.

(4) During the course of this study, a similar approach was recently reported for construction of the ethylidene-piperidine moiety and used to synthesize $( \pm)$-conolidine and $( \pm)$-apparicine: Takanashi, N.; Suzuki, K.; Kitajima, M.; Takayama, H. Tetrahedron Lett. 2016, 57, 375.

(5) For recent reviews, see: (a) Corma, A.; Leyva-Pérez, A.; Sabater, M. J. Chem. Rev. 2011, 111, 1657. (b) Garayalde, D.; Nevado, C. ACS Catal. 2012, 2, 1462. (c) Cera, G.; Bandini, M. Isr. J. Chem. 2013, 53, 848. (d) Nunes dos Santos Comprido, L.; Hashmi, A. S. K. Isr. J. Chem. 2013, 53, 883. (e) Gatineau, D.; Goddard, J.-P.; Mouriès-Mansuy, V.; Fensterbank, L. Isr. J. Chem. 2013, 53, 892. (f) Brand, J. P.; Waser, J. Isr. J. Chem. 2013, 53, 901.

(6) (a) Gorin, D. J.; Dubé, P.; Toste, F. D. J. Am. Chem. Soc. 2006, 128, 14480. (b) Krause, N.; Winter, C. Chem. Rev. 2011, 111, 1994. (c) Ohno, H. Isr. J. Chem. 2013, 53, 869. (d) Qian, D.; Zhang, J. Chem. Rec. 2014, 14, 280. (e) Dorel, R.; Echavarren, A. M. Chem. Rev. 2015, 115, 9028. (f) Pflästerer D.; Hashmi, A. S. K. Chem. Soc. Rev. 2016, 45, 1331.

(7) Naoe, S.; Saito, T.; Uchiyama, M.; Oishi, S.; Fujii, F.; Ohno, H. Org. Lett. 2015, 17, 1774.

(8) Gold(I)-catalyzed reactions of alkyne and silyl enol ether have been reported as powerful strategies for the construction of carbocyclic structures, see: (a) Staben, S. T.; Kennedy-Smith, J. J.; Huang, D.; Corkey, B. K.; LaLonde, R. L.; Toste, F. D. Angew. Chem., Int. Ed. 2006, 45, 5991. (b) Lee, K.; Lee, P. H. Adv. Synth. Catal. 2007, 349, 2092. (c) Minnihan, E. C.; Colletti, S. L.; Toste, F. D.; Shen, H. C. J. Org. Chem. 2007, 72, 6287. (d) Barablé, F.; Bétournay, G.; 
Bellavance, G.; Barriault, L. Org. Lett. 2009, 11, 4236. (e) Kusama, H.; Karibe, Y.; Onizawa, Y.; Iwasawa, N. Angew. Chem., Int. Ed. 2010, 49, 4269. (f) Barabé, F.; Levesque, P.; Korobkov, I.; Barriault, L. Org. Lett. 2011, 13, 5580. (g) Sow, B.; Bellavance, G.; Barabé, F.; Barriault, F. Beilstein J. Org. Chem. 2011, 7, 1007. (h) Brazeau, J.-F.; Zhang, S.; Colomer, I.; Corkey, B. K.; Toste, F. D. J. Am. Chem. Soc. 2012, 134, 2742. (i) Iwai, T.; Okochi, H.; Ito, H.; Sawamura, M. Angew. Chem., Int. Ed. 2013, 52, 4239.

(9) Teichert, J. F.; Zhang, S.; Zijl, A. W. V.; Slaa, J. W.; Minnaard, A. J.; Feringa, B. L. Org. Lett. 2010, 20, 4658.

(10) For a recent review, see: Sindhu, K. S.; Thankachan, A. P.; Sajitha P. S.; Anilkumar, G. Org. Biomol. Chem., 2015, 13, 6891.

(11) Barbazanges, M.; Augé, M.; Moussa, J.; Amouri, H.; Aubert, C.; Desmarets, C.; Fensterbank, L.; Gandon, V.; Malacria, M.; Ollivier, C. Chem. Eur. J. 2011, 17, 13789.

(12) The geometries of 11a and 11b were determined according to the literature: (a) Reich, H. J.; Holtan, R. C.; Borkowsky, S. L. J. Org. Chem. 1987, 52, 312. (b) Reich, H. J.; Holtan, R. C.; Bolm, C. J. Am. Chem. Soc. 1990, 112, 5609.

(13) The geometry of $\mathbf{2 8}$ was confirmed by an NOE between the protons on the indole 3-position and vinylic carbon.

(14) Job, G. E.; Buchwald, S. L. Org. Lett. 2002, 4, 3703.

(15) (a) Jagtap, R. S.; Joshi, N. N. Tetrahedron: Asymmetry 2011, 22, 1861. (b) Gunderson, K. G.; Shapiro, M. J.; Doti, R. A.: Skiles, J. W. Tetrahedron: Asymmetry 1999, 10, 3263.

(16) Liu, G.; Li, J.; Qiu, L.; Liu, L.; Xu, G.; Ma, B.; Sun, J. Org. Biomol. Chem., 2013, 11, 5998.

(17) Johansson, M. J.; Gorin, D. J.; Stabe, S. T.; Toste, F. D. J. Am. Chem. Soc. 2005, 127, 18002.

(18) Melhado, A. D.; Luparia, M.; Toste, F. D. J. Am. Chem. Soc. 2007, 129, 12638.

(19) Hundertmark, T.; Littke, A. F.; Buchwald, S. L.; Fu, G. C. Org. Lett. 2000, 2, 1729.

(20) Naoe, S.; Suzuki, Y.; Hirano, K; Inaba, Y.; Oishi, S.; Fujii, N.; Ohno, H. J. Org. Chem. 2012, $77,4907$. 
(21) Balaraman, K.; Kesava, V. Synthesis 2010, 20, 3461.

(22) Alami, M; Ferri, F. Tetrahedron Lett. 1996, 37, 2763.

(23) Kinoshita, A.; Sakakibara, N.; Mori, M. Tetrahedron 1999, 55, 8155.

(24) Jervis, P. J.; Kariuki, B. M.; Cox, L. R. Org. Lett. 2006, 8, 4649.

(25) Morita, S.; Otsubo, K.; Matsubara, J.; Ohtani, T.; Uchida, M. Tetrahedron: Asymmetry 1995, 6, 245.

(26) Craig, D.; Goldberg, F. W.; Pett, R. W.; Tholen, N. T. H.; White, A. J. P. Chem. Commun. 2013, 49, 9275. 